\title{
Hybrid Network/Satellite-Based Location Estimation and Tracking Systems for Wireless Networks
}

\author{
Po-Hsuan Tseng, Student Member, IEEE, and Kai-Ten Feng, Member, IEEE
}

\begin{abstract}
Mobile location estimation has attracted much attention in recent years. Location algorithms for mobile stations (MSs) can generally be categorized into network- and satellite-based systems. Both types of systems have their advantages and limitations under different environments (i.e., urban or rural area). In this paper, hybrid location estimation schemes, which combine both the satellite- and the network-based signals, are proposed to provide adaptation to various scenarios for location estimation. By exploiting the fusion algorithm, the proposed fusion-based hybrid (FH) architecture integrates the estimation results that are acquired from both the satellite- and the network-based systems. Two different types of signal selection schemes are adopted within the FH architecture: 1) the fixed set of signal inputs approach and 2) the selective set of signal inputs approach. On the other hand, the unified hybrid architecture employs the proposed hybrid signalselection scheme and the hybrid least square estimator, which can conduct location estimation within a selected set of signal sources from the heterogeneous networks. The Kalman filtering technique is exploited in the proposed algorithms to both eliminate the measurement noises and to track the trajectories of the MSs. Numerical results demonstrate that the proposed hybrid location schemes can provide accurate location estimation by adapting themselves to different environments.
\end{abstract}

Index Terms-Angle of arrival (AOA), Global Positioning System (GPS), Kalman filter, mobile location estimation, time difference of arrival (TDOA), time of arrival (TOA).

\section{INTRODUCTION}

W IRELESS location technologies have received a significant amount of attention over the last few decades. The quality of service of the positioning accuracy has been announced after the issue of the emergency 911 (E-911) subscriber safety service [1]. With the assistance of the information from the positioning system, the required performance and objectives for the targeted mobile station (MS) can be achieved with augmented robustness. In recent years, there have been increasing demands for commercial applications to adopt the location information within their system design, e.g., navigation systems, location-based billing, health care

Manuscript received March 20, 2008; revised September 4, 2008 and March 5, 2009. First published September 29, 2009; current version published November 11, 2009. This work was supported in part by the Ministry of Education Aim for the Top University and Elite Research Center Development Program under Contract 95W803C, the National Science Council under Contract 96-2221-E-009-016, the MediaTek Research Center, National Chiao Tung University, and the Universal Scientific Industrial Company, Taiwan. The review of this paper was coordinated by Prof. A. M. Tonello.

The authors are with the Department of Communications Engineering, National Chiao Tung University, Hsinchu 300, Taiwan (e-mail: walker.cm90g@ nctu.edu.tw; ktfeng@mail.nctu.edu.tw).

Color versions of one or more of the figures in this paper are available online at http://ieeexplore.iee.org.

Digital Object Identifier 10.1109/TVT.2009.2023222 systems, intelligent transportation systems [2], and wireless sensor networks [3]. With the emerging interests in locationbased services, location-estimation algorithms with enhanced precision become necessary for these applications under different circumstances.

A variety of wireless location techniques have been studied and investigated [4], [5]. These schemes can be classified into the following two groups: 1) network-based locationestimation algorithms and 2) satellite-based location-estimation algorithms. The representative systems for network-based location techniques include cellular-based networks and the ultrawideband system [6], which adopt time difference of arrival (TDOA) and angle of arrival (AOA) information for location estimation. The TDOA scheme measures the time difference of signals that come from different wireless base stations (BSs), whereas the AOA technique observes the arriving angle of the signal from the MS. The well-adapted technology for the satellite-based location-estimation method is to utilize the Global Positioning System (GPS), which measures the time of arrival (TOA) of the signals that come from different satellites. It has been studied in previous research [7], [8] that the performance of the aforementioned location-estimation techniques varies under different environments. Due to the shortage of signal sources (e.g., in a rural area) or a severe nonline-of-sight (NLOS) situation (e.g., in an urban area), network-based (i.e., TDOA and AOA) methods result in degraded performance for the location determination of the MS [9], [10]. On the other hand, the major problem for the satellite-based method [11] is that the performance is considerably degraded, whereas the satellite signals are severely blocked (e.g., in an urban or an indoor area).

To achieve better accuracy for location estimation, a hybrid approach should be considered to satisfy the requirements under different environments. However, most existing research does not explicitly design location-estimation algorithms that are targeted for heterogeneous circumstances. The assisted GPS (A-GPS) system is considered to be a well-known technique that fuses the network-based signals to enhance the startup performance of a GPS-based positioning system. With the availability of different types of signal sources, the accuracy of location estimation can be improved. The practical system that provides the implementation of the A-GPS system is contributed to the GpsOne [12] chipset solution. Nevertheless, only high-level description is available, which explains how the GpsOne system conducts hybrid location estimation. There is the lack of detailed information regarding the hybrid architecture and performance evaluation for location estimation under different environments. 
The main objective of this paper is to propose hybrid architectures and algorithms for location estimation under heterogeneous networks. Two different types of hybrid location estimation and tracking systems are proposed in this paper. The first scheme, which is called the fusion-based hybrid (FH) architecture, determines the MSs location by combining the separate outcomes from both the network- and the satellitebased techniques (e.g., by integrating the location information from the cellular network and the GPS receiver). Within each of the two separate channels, the two-step least square (LS) method [13] is utilized to estimate the MSs position based on the measurement inputs. The Bayesian inference model [14], [15] is adopted as the fusion mechanism to acquire the final position estimate from both the satellite system and the cellular networks. On the other hand, the unified hybrid (UH) architecture is proposed as the second scheme. The hybrid signal selection (HSS) scheme is proposed to restrict the number of heterogeneous incoming sources based on their signal qualities. Moreover, the hybrid LS (HLS) estimator is employed to provide the location estimation of the MS from the selected signal inputs (i.e., the combination of the TOA, TDOA, and AOA measurements). The source deficiency problem, which may occur within either the cell- or the satellite-based system, can effectively be mitigated by adopting the proposed UH architecture. Furthermore, the Kalman filtering technique [16] is exploited within the two proposed hybrid architectures, which can provide noise smoothing and location tracking as the MS dynamically moves within the network. The performance of the proposed hybrid location-estimation and tracking schemes are evaluated through simulations under different environments.

Based on the implementation perspective, both hybrid architectures can be employed, depending on the flexibility of the hardware unit within an MS. The following two types of signalselection schemes are employed in these hybrid architectures: 1) the fixed set of signal inputs (FSI) method and 2) the selective set of signal inputs (SSI) method. The FSI algorithm can be utilized by a standardized hardware platform, which consists of the GPS receiver and the radio baseband. Note that two FSI are acquired from each of the two channels within the hardware platform. This type of architecture is beneficial for its standardized cost and implementation. On the other hand, the SSI scheme can be employed in an MS through software implementation or flexible hardware design. The scheme can provide both flexible system upgrade and effective location estimation of the MS under different circumstances. Note that both signal-selection schemes can be implemented with the FH architecture, whereas the UH architecture adopts the SSI approach.

The remainder of this paper is organized as follows. Section II presents the system architectures and the modeling of the signal sources for the proposed schemes. The signalselection algorithms that were adopted within the proposed schemes are explained in Section III. Section IV describes the proposed HLS estimator that is associated with the conventional two-step LS method. Section V illustrates the performance evaluation and the implementation assessment of the proposed hybrid schemes. Section VI draws our conclusions.

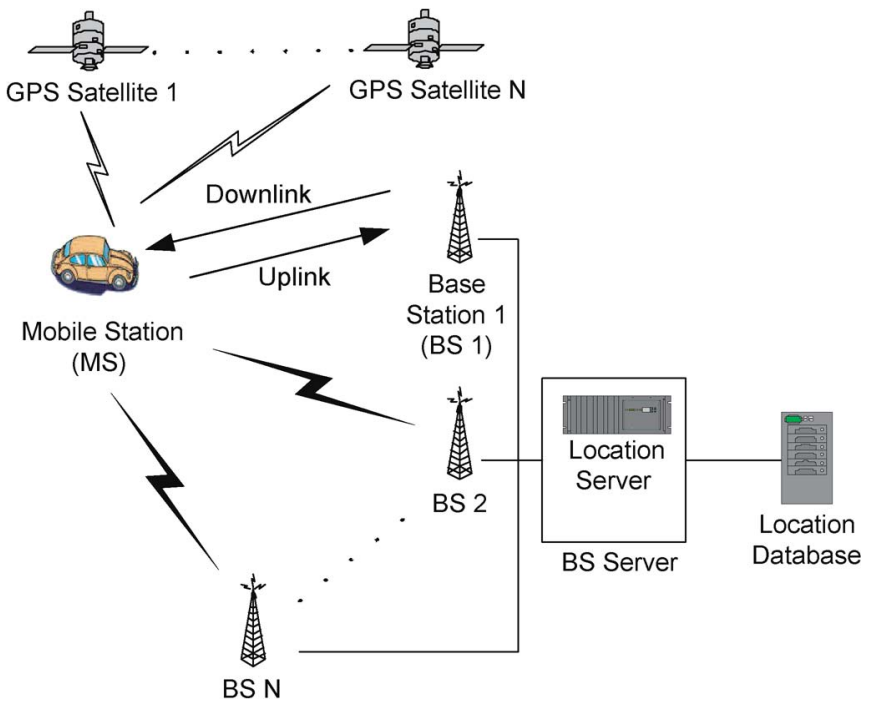

Fig. 1. Schematic of hybrid mobile location estimation.

\section{System ARChitecture AND Modeling}

\section{A. System Architecture}

Fig. 1 shows the schematic of the system architecture for hybrid location estimation. The hybrid system combines the signals that come from both the satellites and the cellular networks. To obtain the TOA measurements from the satellites, it is assumed that the MS should be equipped with a GPS receiver (for the FH scheme) or merely a GPS front end (for the UH scheme). On the other hand, the network-based system adopts the following features from the Third Generation Partnership Project (3GPP) [17] and the WiMax standards [18]: 1) Each BS has a downlink (forward-link) pilot channel that continuously broadcasts its pilot signal to provide timing and phase information for all the MSs in this cellular network; 2) each BS has a dedicated uplink (reverse-link) pilot channel from the MS to provide initial acquisition, time tracking, and power control measurements; 3) each BS is equipped with antenna arrays for adaptive beam steering to facilitate the AOA measurements. As shown in Fig. 1, the TDOA measurements are conducted at the MS by obtaining the signals through the downlink pilot channels from the BSs. The AOA signals are transmitted from the MS to the BSs using the uplink pilot channel. The AOA measurements are performed at the BS using its antenna arrays for 3-D adaptive beam steering. To avoid signal degradation due to the near-far effect, it is assumed that only the home BS provides the capability of the AOA measurements.

As stated in the 3GPP Standard, the location determination of the MS can either be MS based or MS assisted. The choice between these two types of systems depends on the requirement of the communication bandwidth and the computation power of the MS. The FH and UH schemes, as proposed in this paper, can be applied to either the MS-assisted or the MSbased system. The following two sections describe the proposed system architectures based on these two types of systems.

1) Mobile-Assisted System: This type of architecture is suitable for the MS with insufficient computation capability. The left schematic in Fig. 2 illustrates the FH scheme that 

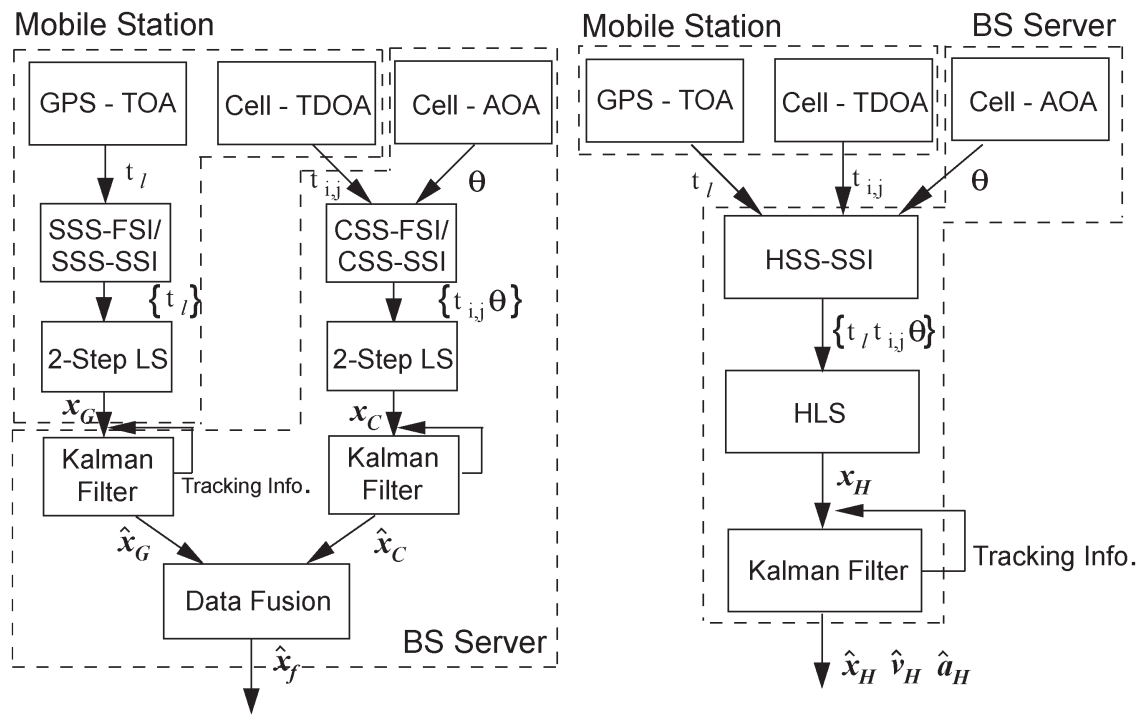

Fig. 2. Mobile-assisted system using (left) the FH architecture and (right) the UH architecture.

is implemented on the MS-assisted positioning system. The following steps describe the procedures of the FH scheme for the MS-assisted system.

1) The GPS-equipped MS receives signals from the satellites and conducts TOA measurement $t_{\ell}$. The GPS receiver either selects five TOA measurements through the satellite-based signal selection with FSI (SSS-FSI) scheme or a flexible number of measurements through the satellite-based signal selection with SSI (SSS-SSI) scheme. The MSs 3-D position (i.e., $\boldsymbol{x}_{G}=$ $\left[\begin{array}{lll}x_{G} & y_{G} & z_{G}\end{array}\right]^{T}$ ) can therefore be estimated using the two-step LS method. On the other hand, the TDOA signals are measured at the MS by obtaining signals from its home BS and the neighboring BSs through the downlink pilot channel.

2) These two sets of information-the location estimation $\boldsymbol{x}_{G}$ and the TDOA measurements $t_{i, j}$-are transmitted back to the home BS through the uplink pilot channel.

3) The AOA measurement $\theta$ is conducted at the home BS by receiving the signals from the MS through the uplink channel.

4) The location server at the home BS performs either the cell-based signal selection for FSI (CSS-FSI) or the cell-based signal selection for SSI (CSS-SSI) scheme from the network-based signal sources, and the two-step LS method is utilized to estimate the 3-D position $\boldsymbol{x}_{C}$ of the MS.

5) The BS location server performs the Kalman filtering technique to smooth out the measurement noises and to track the position data both from the TOA and the TDOA/AOA channels (i.e., $\hat{\boldsymbol{x}}_{G}$ and $\hat{\boldsymbol{x}}_{C}$ ). The detail mechanism of the Kalman filtering formulation is summarized in the Appendix. Note that Kalman tracking will conduct linear prediction (with constant acceleration) to obtain the MSs position, whereas the number of signal inputs becomes insufficient in each of the two channels.

6) Data fusion is performed to merge disparate types of information to enhance position accuracy. The Bayesian inference model [14], [15] is adopted to incorporate both the means $\left(\underline{\hat{x}}_{G}\right.$ and $\left.\underline{\hat{x}}_{C}\right)$ of the filtered estimations $\left(\hat{\boldsymbol{x}}_{G}\right.$ and $\hat{\boldsymbol{x}}_{C}$ ) from the TOA and the TDOA/AOA measurements based on their signal variations, as shown in (1). Note that the parameters $\boldsymbol{\sigma}_{C}=\left(\sigma_{C, x}, \sigma_{C, y}, \sigma_{C, z}\right)$ and $\boldsymbol{\sigma}_{G}=\left(\sigma_{G, x}, \sigma_{G, y}, \sigma_{G, z}\right)$ represent the corresponding standard deviations of $\hat{\boldsymbol{x}}_{C}$ and $\hat{\boldsymbol{x}}_{G}$, respectively. The fused position estimate $\hat{\boldsymbol{x}}_{f}$ of the MS can therefore be obtained as follows:

$$
\begin{aligned}
\hat{\boldsymbol{x}}_{f}= & {\left[\begin{array}{c}
\hat{x}_{f} \\
\hat{y}_{f} \\
\hat{z}_{f}
\end{array}\right]=\left[\begin{array}{ccc}
\frac{\sigma_{G, x}^{2}}{\sigma_{G, x}^{2}+\sigma_{C, x}^{2}} & 0 & 0 \\
0 & \frac{\sigma_{G, y}^{2}}{\sigma_{G, y}^{2}+\sigma_{C, y}^{2}} & 0 \\
0 & 0 & \frac{\sigma_{G, z}^{2}}{\sigma_{G, z}^{2}+\sigma_{C, z}^{2}}
\end{array}\right]\left[\begin{array}{c}
\hat{x}_{C} \\
\frac{\hat{y}}{\hat{z}_{C}}
\end{array}\right] } \\
+ & {\left[\begin{array}{ccc}
\frac{\sigma_{C, x}^{2}}{\sigma_{G, x}^{2}+\sigma_{C, x}^{2}} & 0 & 0 \\
0 & \frac{\sigma_{C, y}^{2}}{\sigma_{G, y}^{2}+\sigma_{C, y}^{2}} & 0 \\
0 & 0 & \frac{\sigma_{C, z}^{2}}{\sigma_{G, z}^{2}+\sigma_{C, z}^{2}}
\end{array}\right]\left[\begin{array}{c}
\underline{\hat{x}}_{G} \\
\frac{\hat{y}}{\hat{z}_{G}}
\end{array}\right] . }
\end{aligned}
$$

The right schematic in Fig. 2 shows the proposed UH scheme that is adopted on the MS-assisted system. Compared with the FH scheme, one of the major differences in the UH architecture is that the TOA measurements $t_{\ell}$ (obtained through the GPS front end within the MS) are transmitted to the BS instead of the estimated MSs position $\boldsymbol{x}_{G}$. After acquiring the three different types of signal inputs (i.e., the TOA, the TDOA, and the AOA measurements), the location server at the home BS will start to perform signal selection through the proposed HSS with SSI (HSS-SSI) scheme. The selection scheme will guarantee the availability of sufficient signal inputs for the HLS estimator and will also eliminate severely interfered signals. The HLS estimator will therefore be conducted to combine the various types of measurement inputs for the estimation of the MSs 3-D position $\boldsymbol{x}_{H}$. Moreover, the Kalman filtering technique is employed to provide consistent location tracking and smoothing $\left(\hat{\boldsymbol{x}}_{H}\right)$ under a wide range of environments. 

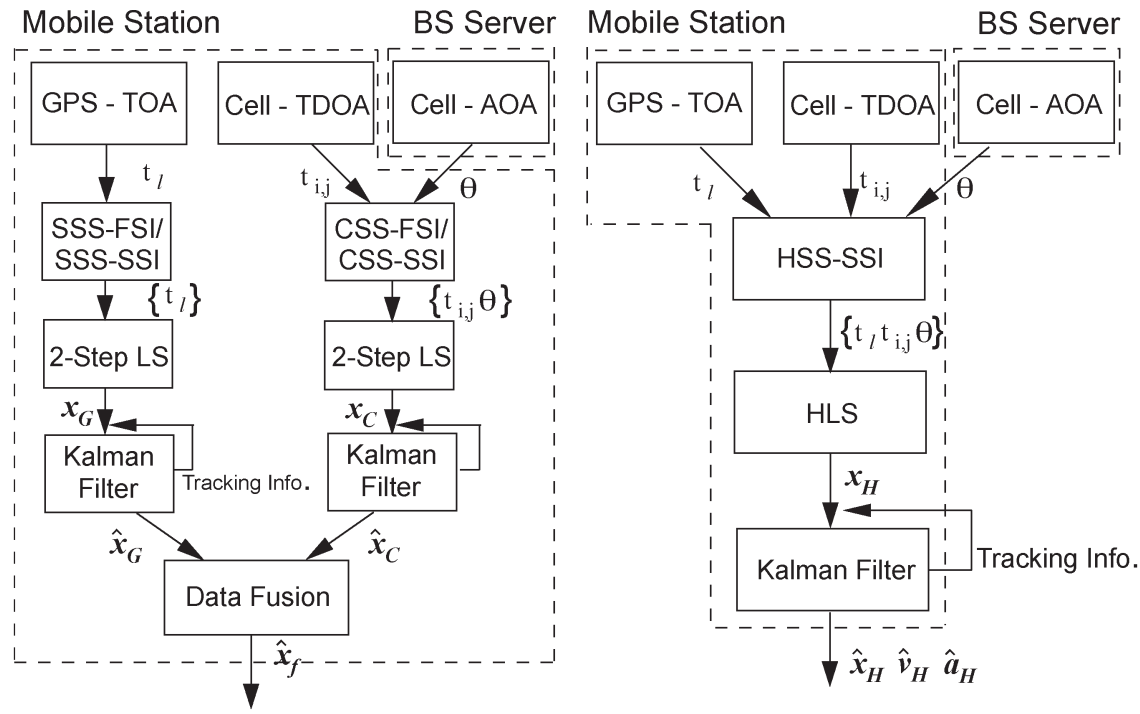

Fig. 3. Mobile-based system using the (left) FH architecture and (right) the UH architecture.

2) Mobile-Based System: This type of architecture is suitable for the MS that possesses adequate computation capability. The left schematic in Fig. 3 shows the proposed FH scheme for the MS-based positioning system. Compared with the left diagram in Fig. 2, the AOA measurement $\theta$ is obtained from the home BS and is transmitted to the MS through the downlink pilot channel. All the remaining functionalities (i.e., signal selection, location estimation, Kalman filtering, and data fusion) are performed at the MS to obtain the final location estimate $\hat{\boldsymbol{x}}_{f}$. On the other hand, the proposed UH scheme for the MS-based positioning system is illustrated in the right diagram in Fig. 3. Similarly, the only function that was conducted by the BS server is to measure the AOA signal $\theta$ and is consequently transmitted back to the MS through the downlink pilot channel. All the other tasks that are accomplished by the MS can be referred to the UH method for the MS-assisted system (as shown in the right diagram in Fig. 2), except those that are performed within the MS in this case.

\section{B. Mathematical Modeling of Signal Inputs}

In this section, the mathematical models for the TOA, TDOA, and AOA measurements are presented. The 3-D coordinates of the MS are exploited in the proposed hybrid location-estimation schemes. The 3-D pseudorange measurement between the MS and the satellites can be obtained by calculating the TOA measurement $t_{\ell}$ from the GPS system by

$$
r_{\ell}=c \cdot t_{\ell}=\zeta_{\ell}+n_{\ell}+\rho_{\ell}+b, \quad \ell=1,2, \ldots N
$$

where $c$ is the speed of light, and $r_{\ell}$ represents the relative distance between the MS and the $\ell$ th satellite, which is contaminated with the TOA measurement noise $n_{\ell}$ and the satelliteinduced error $\rho_{\ell}$. Note that $\rho_{\ell}$ denotes the combined noises other than the measurement noise, e.g., the delays from the ionosphere, the troposphere, and the multipath effect. Moreover, parameter $b$, which is considered identical to all signal sources, indicates the clock bias that is caused by the clock off- set between the MS and the satellite. The noiseless relative distance $\zeta_{\ell}$ between the MS and the $\ell$ th satellite can be obtained as

$$
\zeta_{\ell}=\left[\left(x-x_{\ell}\right)^{2}+\left(y-y_{\ell}\right)^{2}+\left(z-z_{\ell}\right)^{2}\right]^{\frac{1}{2}}
$$

where $(x, y, z)$ represents the MSs position, and $\left(x_{\ell}, y_{\ell}, z_{\ell}\right)$ is the location of the $\ell$ th satellite. On the other hand, the cellularbased 3-D relative distance $r_{i, j}$ can be obtained by computing the TDOA measurement $t_{i, j}$, which is the time difference between the MS with respect to the $i$ th and the $j$ th BSs, as

$$
r_{i, j}=c \cdot t_{i, j}=\left(\zeta_{i}-\zeta_{j}\right)+\left(n_{i}-n_{j}\right)+\left(n_{n l, i}-n_{n l, j}\right)
$$

where $n_{i}$ and $n_{j}$ represent the measurement noises from the MS to the $i$ th and the $j$ th BSs, respectively. The NLOS errors $n_{n l, i}$ and $n_{n l, j}$ are considered to be nonnegative values between the MS and the $i$ th and the $j$ th BSs. Moreover, the horizontal and the vertical AOA measurements that are available from the home BS can be obtained as

$$
\begin{aligned}
& \theta=\tan ^{-1}\left(\frac{y-y_{1}}{x-x_{1}}\right)+n_{\theta} \\
& \phi=\tan ^{-1}\left(\frac{z-z_{1}}{\left[\left(x-x_{1}\right)^{2}+\left(y-y_{1}\right)^{2}\right]^{\frac{1}{2}}}\right)+n_{\phi}
\end{aligned}
$$

where $\theta$ and $\phi$ represent the horizontal (azimuth) and the vertical (elevation) angles between the MS and its home BS (i.e., $\left.\mathrm{BS}_{1}\right)$. Note that $\left(x_{1}, y_{1}, z_{1}\right)$ corresponds to the coordinate of $\mathrm{BS}_{1}$. Parameters $n_{\theta}$ and $n_{\phi}$ are the combined noises that are associated with angles $\theta$ and $\phi$.

\section{Signal Selection Schemes for the Proposed HYBRID ARCHITECTURES}

Conventional studies [19] indicate that all of the signal inputs should be utilized for location estimation to achieve better performance under the situation in which the measurements are line-of-sight (LOS) signals. In particular, the Cramér-Rao lower bound (i.e., the error lower bound) under an LOS 
environment monotonically decreases as the number of the BSs is augmented. However, in realistic environments, it is expected to encounter situations with different error distributions [20][22], e.g., with an environment that contains both the NLOS and LOS measurements. In other words, provided that the NLOS measurement exists, the strategy of adopting all of the signal measurements will not be considered an optimal method. As a result, it is required to exploit a signal-selection scheme among the various signal sources.

Signal-selection schemes are considered the postprocessing approach after the front-end hardware transceiver has prefiltered the input signals, e.g., by eliminating the interferences from the fading effects. Different types of signal selection algorithms have been proposed. It has been studied [23], [24] as an NP-hard problem to obtain the optimal set of signal sources (out of exhaustive search on the sample space) to achieve the minimum estimation error for the MSs position. Therefore, approximate solutions for achieving the optimal set of signal sources are proposed in the next sections. The selection strategy for both the FSI and SSI schemes are proposed based on different criteria under the satellite-based (see Section III-A) and the cell-based (see Section III-B) networks, respectively. In Section III-C, the SSI scheme is exploited to sort out the available sources based on their signal qualities under heterogeneous environments. Note that choosing between FSI and SSI depends on the tradeoffs between the hardware cost and the implementation flexibility. Without severe degradation on the estimation accuracy, the proposed FSI scheme can be implemented in a standardized hardware platform to reduce hardware cost and increase computation speed. On the other hand, by considering software implementation, the proposed SSI scheme can achieve better estimation accuracy without being constrained by a fixed number of signal sources.

\section{A. SSS Scheme}

The SSS-FSI and SSS-SSI schemes within the FH architecture (as shown in the left diagrams in Figs. 2 and 3) are presented in this section. The procedure of the SSS approach is to first utilize the receiver autonomous integrity monitoring (RAIM) [25] technique for the elimination of possible faulty satellites. The remaining satellites will further be distinguished with either good or bad signal quality according to both their elevation angles and signal-to-noise ratio (SNR) values. Moreover, in the SSS-FSI scheme, the total number of signals is further constrained to be a fixed number for ease of implementation. In general, four measurement inputs will be sufficient for the estimation of four state variables. However, due to the nonlinear behavior (i.e., terms with square root) associated with the equations, additional TOA measurement will be required for the closed-form location estimator [26], [27], e.g., the two-step LS estimator. To achieve higher estimation accuracy, there is also literature [11] that seeks for solutions with more available signal sources. Therefore, the minimum number of the satellites that was required for the SSS-FSI scheme is chosen to be five.

Several factors [26] can degrade the satellite signals and consequently affect the accuracy for MSs location-estimation algorithm, e.g., the number of visible satellites and the satellite geometry. To effectively consider these inferior effects to the accuracy for location estimation, three parameters are considered in the proposed SSS scheme: 1) the elevation angle of satellite; 2) the SNR value [28]; and 3) the Geometric Dilution of Position (GDOP) metric. In general, severe multipath effect will be incurred with comparably smaller satellite elevation angle, whereas the SNR value can be utilized as an index to remove weak signal sources. Meanwhile, note that GDOP [29] is a well-adopted metric to justify the accuracy of location estimation based on the geometric layouts between the MS and its associated satellites. Furthermore, the RAIM technique is also adopted to examine the integrity of GPS pseudorange measurements. Within the scheme of RAIM fault detection and exclusion (RAIM-FDE) [30], the GPS receiver can both detect and isolate the erroneous channels from those that can be utilized for location estimation.

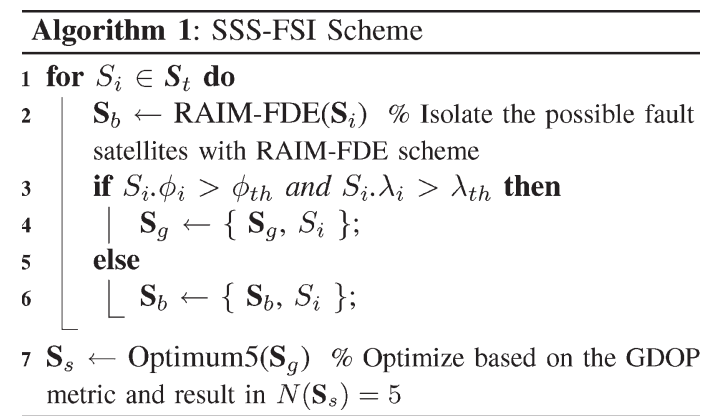

The proposed SSS-FSI scheme is first illustrated in Algorithm 1, whereas the difference between the SSS-SSI and SSS-FSI schemes will be described afterward. In Algorithm 1, the entire set of available satellites is defined as $\mathbf{S}_{t}=$ $\left\{\mathbf{S}_{g}, \mathbf{S}_{b}\right\}=\left\{S_{i} \mid \forall i, 1 \leq i \leq n\right\}$, where $\mathbf{S}_{g}$ and $\mathbf{S}_{b}$ are represented as the sets of satellites with good and bad signal qualities. $\mathbf{S}_{s} \in \mathbf{S}_{t}$ is the satellite set that is selected by the SSS-FSI scheme. $N(\Phi)$ denotes the number of elements within the $\Phi$ set. Initially, the proposed scheme utilizes the RAIM-FDE scheme to detect and isolate the satellites $S_{i}$ that are detected to cause the fault. Note that the RAIM-FDE technique will only eliminate those satellites that fail to pass the detection. The proposed SSS-FSI scheme continues to construct the remaining $\mathbf{S}_{g}$ set by verifying if the elevation angle $\left(\phi_{i}\right)$ and the SNR value $\left(\lambda_{i}\right)$ of the satellite $S_{i}$ are larger than their prespecified thresholds $\phi_{t h}$ and $\lambda_{t h}$. Furthermore, the minimal required number of satellites for the proposed SSS-FSI scheme can be acquired with the exploitation of the Optimum5 scheme, as in Algorithm 1. Basically, this technique is adopted from [31] to obtain the optimal set of satellites $\mathbf{S}_{s}$ (which is chosen as five) based on the minimal GDOP criterion. However, the computation of the minimum GDOP value involves a series of matrix operations, which is considered inapplicable for implementation. To compute the GDOP value in a real-time manner, a matrix inversion lemma is utilized to approximate the complex matrix calculation into a recursive computation with single-order operations, i.e., to reduce the computation cost from $O\left(n^{3}\right)$ to $O(n)$. Moreover, by adopting the revolvingdoor method, the previous calculation can further be reused instead of conducting an entire matrix calculation. With recursive 
computation, it is also feasible to employ this scheme under situations in which visible satellites change from time to time.

On the other hand, compared with the SSS-FSI approach, the proposed SSS-SSI scheme removes the constraint that the total the number of satellites in the selected set $\mathbf{S}_{s}$ should be five. In other words, the Optimum5 function, as implemented in Line 7 of Algorithm 1, is removed, which consequently results in $\mathbf{S}_{s}=\mathbf{S}_{g}$. Furthermore, note that the SSS-FSI and SSS-SSI schemes become the same algorithm, provided that $N\left(\mathbf{S}_{g}\right)$ is smaller or equal to the number of five. In Section V, the performance comparisons between these two signal-selection schemes will further be evaluated from both the computation cost and estimation accuracy.

\section{B. CSS Scheme}

This section describes the CSS-FSI and CSS-SSI schemes within the FH architecture (as shown in the left diagrams in Figs. 2 and 3). As indicated in (4), the range measurement $r_{i, j}$ is contaminated with the measurement errors $\left(n_{i}\right.$ and $\left.n_{j}\right)$ and the NLOS errors $\left(n_{n l, i}\right.$ and $\left.n_{n l, j}\right)$. The NLOS error, which represents the additional propagation time (i.e., with a positive value), has been observed as a dominate issue for the timebased location estimation [20]-[22], [32]-[34] and is hard to remove from the front-end signal processing. It has been investigated that the NLOS error can exceed $589 \mathrm{~m}$ within the IS-95 code-division multiple access networks [33]. The large bias in the range measurement will severely decrease the accuracy of the network-based location-estimation algorithms. Consequently, the proposed CSS scheme will primarily focus on selecting the measurement inputs that are less corrupted by the NLOS errors.

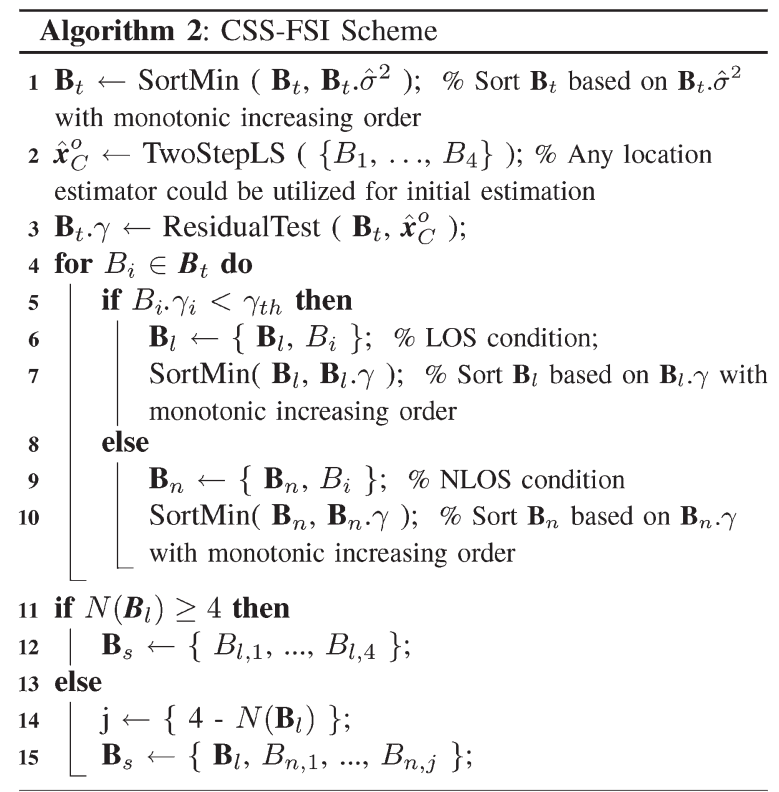

The concept of the CSS-FSI approach will first be explained in Algorithm 2, whereas the difference between the CSS-SSI and CSS-FSI schemes will be described afterward. In Algorithm 2, the set of available BSs is defined as $\mathbf{B}_{t}=$ $\left\{\mathbf{B}_{l}, \mathbf{B}_{n}\right\}=\left\{B_{i} \mid \forall i, 1 \leq i \leq m\right\}$, where $\mathbf{B}_{l}$ and $\mathbf{B}_{n}$ are represented as the sets of BSs that belong to the LOS and NLOS measurements, respectively. $\mathbf{B}_{s} \in \mathbf{B}_{t}$ is denoted as the BS set that is selected by the proposed CSS-FSI scheme. Initially, the set of available BSs $\mathbf{B}_{t}$ is sorted with monotonic increasing order based on the signal variance from its corresponding BS. Based on the selected four BSs (i.e., $B_{1}-B_{4}$ ) with comparably smaller signal variances, an initial MSs estimated position $\hat{\boldsymbol{x}}_{C}^{o}$ can be acquired by adopting a conventional location-estimation method, e.g., the two-step LS method [35] in our case. Note that four measurement inputs, which result in three TDOA signals [as in (4)], will compose the minimal required number of BSs for the estimation of the MSs 3-D position.

To identify the NLOS errors from the TDOA range measurements, a residual-test that was modified based on [20] is exploited after the initial location estimation. Note that the main concept of the residual test is to distinguish potential NLOS measurement based on the existence of LOS signal sources for location estimation. The outcome of the residual-test $\gamma_{i}$ for the BS $B_{i}$ can be represented as

$$
\begin{aligned}
\gamma_{i} & =F\left(r_{i, j}-d_{i, j}\left(\hat{\boldsymbol{x}}_{C}^{o}\right) \mid \hat{n}_{n l, i}=0, \hat{n}_{n l, j}=0\right) \\
& =\frac{1}{2}+\frac{1}{2} \operatorname{erf}\left(\frac{\left(\mathrm{r}_{\mathrm{i}, \mathrm{j}}-\mathrm{d}_{\mathrm{i}, \mathrm{j}}\left(\hat{\boldsymbol{x}}_{\mathrm{C}}^{\mathrm{o}}\right)\right)}{\sqrt{2} \hat{\sigma}_{\mathrm{i}}}\right)
\end{aligned}
$$

where $d_{i, j}\left(\hat{\boldsymbol{x}}_{C}^{o}\right)$ denotes the difference between the distances (i.e., $d_{i}\left(\hat{\boldsymbol{x}}_{C}^{o}\right)$ and $d_{j}\left(\hat{\boldsymbol{x}}_{C}^{o}\right)$ ) from the initial estimated position $\hat{\boldsymbol{x}}_{C}^{o}$ to the positions of $B_{i}$ and $B_{j}$. The main concept of the residual test in (7) is to determine the residual $\gamma_{i}$ based on the observation from the difference between the measured and the reference distances. The noiseless relative distance $\zeta_{i, j}$ is considered unattainable; thus, $d_{i, j}\left(\hat{\boldsymbol{x}}_{C}^{o}\right)$ is utilized to approximately represent the reference relative distance. The error function lies between $[-1,1]$; thus, the outcome of the residual-test $\gamma_{i}$ will be within the range of $[0,1]$. Provided that the measurement $r_{i, j}$ contains the NLOS error, the measurement distance $r_{i, j}$ should be larger than the reference distance $d_{i, j}\left(\hat{\boldsymbol{x}}_{C}^{o}\right)$. Note that, even with the possibility of encountering a poor initial estimate $\hat{\boldsymbol{x}}_{C}^{o}, r_{i, j}$ will still be larger than $d_{i, j}\left(\hat{\boldsymbol{x}}_{C}^{o}\right)$, because $d_{i, j}\left(\hat{\boldsymbol{x}}_{C}^{o}\right)$ should always fall within an area that is confined by its corresponding BSs. Consequently, the residual value $\gamma_{i}$ will result in a larger value, i.e., in general, it lies between 0.5 and 1 . On the other hand, the $\gamma_{i}$ value will reside between 0 and 0.5 under situations with LOS environments. The $\gamma_{i}$ value for each $B_{i}$ will be compared with a predetermined threshold $\gamma_{t h}$, which is assigned around 0.5 , to identify the existence of the NLOS error from the LOS measurement inputs. After acquiring both the $\mathbf{B}_{l}$ and $\mathbf{B}_{n}$ sets, the CSS-FSI scheme will obtain the set with the minimal required number of BSs (i.e., $\mathbf{B}_{s}$ ) that is associated with comparably smaller NLOS errors. Note that the residual test can become ineffective under the condition that all the signals in the $\mathbf{B}_{t}$ set are with NLOS errors. In such a case, the NLOS BSs within the $\mathbf{B}_{n}$ set will be selected for location estimation, as illustrated in Algorithm 2. In the performance evaluation section (i.e., Section V), both the identification rate for NLOS signals and the estimation accuracy that incorporates the potential failure of residual test are considered in the simulations. 
Similarly, compared with the CSS-FSI approach, the proposed CSS-SSI scheme removes the constraint of a fixed number of measurement inputs. All the LOS signal inputs will be utilized in the CSS-SSI scheme for location estimation of the MS. Performance comparison between these two selection schemes will be addressed in Section V.

\section{HSS-SSI Scheme}

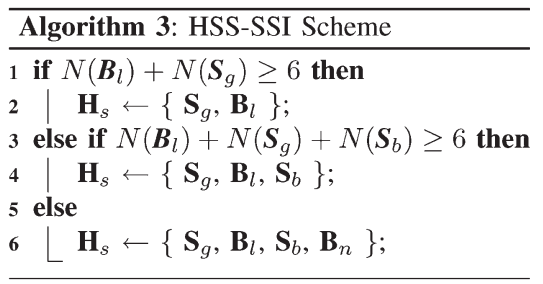

As shown in the right diagrams in Figs. 2 and 3, the HSS scheme for the UH is presented in this section. Unlike the previous two selection schemes for the FSI case with a fixed number of signal inputs (i.e., $N\left(\mathbf{S}_{s}\right)=5$, and $N\left(\mathbf{B}_{s}\right)=4$ ), the number of selected signal sources (i.e., $N\left(\mathbf{H}_{s}\right)$ ) is flexibly within the HSS-SSI scheme, i.e., $6 \leq N\left(\mathbf{H}_{s}\right) \leq N\left(\mathbf{S}_{t}\right)+N\left(\mathbf{B}_{t}\right)$. Note that the smallest number for the scheme (i.e., $N\left(\mathbf{H}_{s}\right)=6$ ) is considered the minimal number of input requirements by using the HLS estimator, which will be addressed in Section IV-B. The objective of the proposed HSS-SSI scheme is primarily based on choosing the sources with better signal qualities. As illustrated in Algorithm 3, the selection strategy is based on prioritizing the following three categories: 1) the satellites with good signal quality $\mathbf{S}_{g}$ and the BSs with LOS measurement $\mathbf{B}_{l}$; 2) the satellite with bad signal quality $\mathbf{S}_{b}$; and 3 ) the BSs with NLOS measurement $\mathbf{B}_{n}$. Categories 2 and 3 will not be chosen, as long as there are a sufficient number of signal inputs available in Category 1. Moreover, the number of signal inputs that was obtained from the HSS-SSI scheme will not be confined as that in the FSI scheme.

\section{Hybrid Least SQuares LOCATION-ESTIMATION ALGORITHM}

In this section, the HLS location-estimation scheme is proposed for the UH system, whereas the two-step LS method that was extended from the conventional algorithm will be utilized in the FH system.

\section{A. Two-Step LS Location Algorithm}

To provide computational efficiency, closed-form location schemes are suggested for real-time applications, e.g., the LS-based estimators. In particular, the two-step LS estimator [13] is an approximate realization of the maximum-likelihood estimator and does not require iterative processes. The concept of the two-step LS scheme is to acquire an intermediate location estimate in the first step, with the definition of a new variable, i.e., $\beta=x^{2}+y^{2}+z^{2}$, which is a nonlinear term and is mathematically related to the MSs position. At this stage, this variable is assumed to be uncorrelated to the MSs position (i.e., $x, y$, and $z$ ). This variable assignment effectively transforms the nonlinear equations for location estimation into a set of linear equations, which can directly be solved by the weighted LS method. The second step of the two-step LS scheme primarily associates the known relationship to form the weighted LS formulation between the BSs coordinate, the intermediate variable (i.e., $\beta$ ), and the MSs position, which was originally assumed to be uncorrelated in the first step. The information redundancy from the first-step LS is adopted to update the MS coordinate. An improved location estimation (i.e., $x, y$, and $z$ ) can therefore be obtained after the adjustment from the second step.

The two-step LS estimator was adopted to solve the location estimation problem from the TOA [8], TDOA [13], and TDOA/AOA measurements [35]. In addition to estimating the 2-D position of the MS as in the previous research, the two-step LS method is applied in the proposed FH system to calculate the 3-D location of the MS. In particular, the clock bias is also included within the FH system. The following two sections describe the 3-D TOA location estimation for the satellite-based system and the 3-D TDOA/AOA location-estimation algorithm for the cellular network.

1) Three-Dimensional TOA Location Estimation: To estimate the MSs 3-D position (i.e., $x, y$, and $z$ ) and the clock bias (i.e., $b$ ) within the two-step LS formulation from the TOA measurements, it is assumed that signals that come from at least five satellites are available. The 3-D TOA measurements, as described in (2), can be rewritten in matrix form as $\mathbf{M} \boldsymbol{x}=\mathbf{J}$, where

$$
\begin{aligned}
\mathbf{M} & =\left[\begin{array}{ccccc}
-2 x_{1} & -2 y_{1} & -2 z_{1} & 2 r_{1} & 1 \\
-2 x_{2} & -2 y_{2} & -2 z_{2} & 2 r_{2} & 1 \\
\cdot & \cdot & \cdot & \cdot & \cdot \\
-2 x_{N} & -2 y_{N} & -2 z_{N} & 2 r_{N} & 1
\end{array}\right] \\
\mathbf{J} & =\left[\begin{array}{l}
r_{1}^{2}-\kappa_{1} \\
r_{2}^{2}-\kappa_{2} \\
\cdot \\
r_{N}^{2}-\kappa_{N}
\end{array}\right]
\end{aligned}
$$

with $\boldsymbol{x}=\left[\begin{array}{lllll}x & y & z & b & \beta\end{array}\right]^{T}$. Note that $\beta=x^{2}+y^{2}+z^{2}-$ $b^{2}$ and that $\kappa_{\ell}=x_{\ell}^{2}+y_{\ell}^{2}+z_{\ell}^{2}$. The concept of the two-step LS method is to acquire an intermediate location estimate in the first step by assuming that $(x, y, z, b)$ and $\beta$ are not correlated. The second step of the method releases this assumption by adjusting the intermediate result to obtain an improved location estimate, i.e., $\boldsymbol{x}_{G}=\left[\begin{array}{lll}x_{G} & y_{G} & z_{G}\end{array}\right]^{T}$. The details of the twostep LS method can be found in [8].

2) Three-Dimensional TDOA/AOA Location Estimation: To solve for the two-step LS problem for the cellular-based system, the home BS should provide both the TOA and AOA measurements, whereas three additional TOA measurements are assumed to be obtainable from other BSs. The 3-D TDOA and 
AOA measurements, as in (4)-(6), can also be rewritten in the form of $\mathbf{M} \boldsymbol{x}=\mathbf{J}$, where the matrices $\mathbf{M}$ and $\mathbf{J}$ become

$$
\begin{aligned}
\mathbf{M} & =-2 \cdot\left[\begin{array}{cccc}
x_{2}-x_{1} & y_{2}-y_{1} & z_{2}-z_{1} & r_{2,1} \\
x_{3}-x_{1} & y_{3}-y_{1} & z_{3}-z_{1} & r_{3,1} \\
\vdots & \ldots & \ldots & \vdots \\
x_{N}-x_{1} & y_{N}-y_{1} & z_{N}-z_{1} & r_{N, 1} \\
-\sin \theta & \cos \theta & 0 & 0 \\
0 & 0 & \cos \phi & -\cos \phi \sin \phi
\end{array}\right] \\
\mathbf{J} & =\left[\begin{array}{c}
r_{2,1}^{2}-\kappa_{2}+\kappa_{1} \\
r_{3,1}^{2}-\kappa_{3}+\kappa_{1} \\
\cdots \\
r_{N, 1}^{2}-\kappa_{N}+\kappa_{1} \\
2 x_{1} \sin \theta-2 y_{1} \cos \theta \\
-2 z_{1} \cos \phi
\end{array}\right]
\end{aligned}
$$

with $\boldsymbol{x}=\left[\begin{array}{llll}x & y & z & r_{1}\end{array}\right]^{T}$. In both matrices, the AOA components are computed based on the geometric approximation, as described in [35]. The two-step LS method can therefore be applied to obtain the location estimates from the TDOA/AOA measurements. Equation (10), shown at the bottom of the page, holds.

\section{B. HLS Location-Estimation Algorithm}

In the UH system, the MS can utilize different types of signal sources to acquire the location information. The HLS location estimator is proposed in this section to facilitate the MSs location estimation with heterogeneous signal inputs. In particular, note that the combination of the TOA and TDOA/AOA channels within the two-step LS formulation is nontrivial. Within the formulation of the conventional two-step LS location algorithm, the variables that were introduced in the TOA-based scheme include $x, y, z, b$, and $\beta$ (as discussed in Section IV-A1), whereas the variables for the TDOA/AOA-based approach comprise $x, y, z$, and $r_{1}$ (as discussed in Section IV-A2). To eliminate the nonlinear terms in both schemes, the intermediate variables $\beta$ and $r_{1}$ are utilized, along with the desired estimated parameters $x, y, z$, and $b$. Consequently, the total number of required measurement inputs for the proposed HLS estimator will become six, i.e., for the estimation of the variables $\beta, r_{1}$, $x, y, z$, and $b$. Due to the resulting two intermediate variables, an additional step will be required within the HLS location estimator.

Within the TOA-based two-step LS formation, the circular equation $r_{\ell}=\zeta_{\ell}+b$ for the TOA measurement $r_{\ell}$ is represented as

$$
\left(r_{\ell}-b\right)^{2}=\zeta_{\ell}^{2}=\left(x_{\ell}-x\right)^{2}+\left(y_{\ell}-y\right)^{2}+\left(z_{\ell}-z\right)^{2}
$$

which can further be expanded as

$$
r_{\ell}^{2}=\beta-2 x_{\ell} \cdot x-2 y_{\ell} \cdot y-2 z_{\ell} \cdot z+2 r_{\ell} \cdot b+\kappa_{\ell}
$$

with $\beta=x^{2}+y^{2}+z^{2}-b^{2}$ and $\kappa_{\ell}=x_{\ell}^{2}+y_{\ell}^{2}+z_{\ell}^{2}$. The first step of the two-step LS scheme is to solve the weighted LS problem through the "linear" equation with variables $(x, y, z$, $b$, and $\beta$ ). On the other hand, the TDOA/AOA-based two-step LS problem considers the hyperbolic equation for the TDOA measurement $r_{i, 1}$, considering the $i$ th $\mathrm{BS}$ with respect to the home BS, as

$$
r_{i, 1}^{2}=2 r_{i, 1} \cdot r_{1}-2 x_{i, 1} \cdot x-2 y_{i, 1} \cdot y-2 z_{i, 1} \cdot z+\left(\kappa_{i}-\kappa_{1}\right)
$$

where the four variables $\left(x, y, z, r_{1}\right)$ are considered to be solved within the two-step LS formulation. Note that the variables $r_{1}$ and $\beta$ are functions of the $x, y$, and $z$ variables. By combining (12) and (13), the elements within the matrix formulation (i.e., $\mathbf{M}_{1} \boldsymbol{x}_{H}^{(1)}=\mathbf{J}_{1}$ ) for the first step of the proposed HLS estimator can be obtained as in (10) ${ }^{1}$ and (14) as follows:

$$
\mathbf{J}_{1}=\left[\begin{array}{c}
\mathbf{J}_{\mathrm{TD}}^{N\left(\mathbf{B}_{l}\right) \times 1} \\
\mathbf{J}_{\mathrm{AOA}}^{2 \times 1} \\
\mathbf{J}_{\mathrm{TOA}}^{N\left(\mathbf{S}_{g}\right) \times 1}
\end{array}\right]=\left[\begin{array}{c}
r_{2,1}^{2}-\kappa_{2}+\kappa_{1} \\
r_{3,1}^{2}-\kappa_{3}+\kappa_{1} \\
\cdots \\
r_{N\left(\mathbf{B}_{l}\right), 1}^{2}-\kappa_{N\left(\mathbf{B}_{l}\right)}+\kappa_{1} \\
-x_{1} \sin \theta+y_{1} \cos \theta \\
z_{1} \cos \phi \\
\tilde{r}_{1}^{2}-\tilde{\kappa}_{1} \\
\cdots \\
\tilde{r}_{N\left(\mathbf{s}_{g}\right)}^{2}-\tilde{\kappa}_{N\left(\mathbf{S}_{g}\right)}
\end{array}\right]
$$

${ }^{1}$ For ease of notation, variables with a tilde are denoted for the satellite-based variables, e.g., $\tilde{r}_{1}$, whereas variables without a tilde (e.g., $\left.r_{1}\right)$ are utilized as network-based variables.

$$
\mathbf{M}_{1}=\left[\begin{array}{c}
\mathbf{M}_{\mathrm{TDOA}}^{N\left(\mathbf{B}_{l}\right) \times 6} \\
\mathbf{M}_{\mathrm{AOA}}^{2 \times 6} \\
\mathbf{M}_{\mathrm{TOA}}^{N\left(\mathbf{S}_{g}\right) \times 6}
\end{array}\right]=\left[\begin{array}{cccccc}
-2\left(x_{2}-x_{1}\right) & -2\left(y_{2}-y_{1}\right) & -2\left(z_{2}-z_{1}\right) & 0 & 0 & -2 r_{2,1} \\
-2\left(x_{3}-x_{1}\right) & -2\left(y_{3}-y_{1}\right) & -2\left(z_{3}-z_{1}\right) & 0 & 0 & -2 r_{3,1} \\
\vdots & \ldots & \ldots & \ldots & \ldots & \vdots \\
-2\left(x_{N\left(\mathbf{B}_{l}\right)}-x_{1}\right) & -2\left(y_{N\left(\mathbf{B}_{l}\right)}-y_{1}\right) & -2\left(z_{N\left(\mathbf{B}_{l}\right)}-z_{1}\right) & 0 & 0 & -2 r_{N\left(\mathbf{B}_{l}\right), 1} \\
-\sin \theta & \cos \theta & 0 & 0 & 0 & 0 \\
0 & 0 & \cos \phi & 0 & 0 & -\cos \phi \sin \phi \\
-2 \tilde{x}_{1} & -2 \tilde{y}_{1} & -2 \tilde{z}_{1} & 2 \tilde{r}_{1} & 1 & 0 \\
\vdots & \ldots & \ldots & \ldots & \ldots & \vdots \\
-2 \tilde{x}_{N\left(\mathbf{S}_{g}\right)} & -2 \tilde{y}_{N\left(\mathbf{S}_{g}\right)} & -2 \tilde{z}_{N\left(\mathbf{S}_{g}\right)} & 2 \tilde{r}_{N\left(\mathbf{S}_{g}\right)} & 1 & 0
\end{array}\right]
$$




$$
\begin{aligned}
& \text { with } \boldsymbol{x}_{H}^{(1)}=\left[\begin{array}{llllll}
x^{(1)} & y^{(1)} & z^{(1)} & b^{(1)} & \beta^{(1)} & r_{1}^{(1)}
\end{array}\right]^{T} \text { as } \\
& \boldsymbol{x}_{H}^{(1)}=\left(\mathbf{M}_{1}^{T} \boldsymbol{\Psi}_{1}^{-1} \mathbf{M}_{1}\right)^{-1} \mathbf{M}_{1}^{T} \boldsymbol{\Psi}_{1}^{-1} \mathbf{J}_{1} .
\end{aligned}
$$

The error of the first step estimation is obtained as $\psi_{1}=$ $\mathbf{M}_{1} \boldsymbol{x}_{H}^{(1)}-\mathbf{J}_{1}=2 c \mathbf{B}_{1} \mathbf{n}_{1}+c^{2} \mathbf{n}_{1}^{2}$; thus, the weighting matrix $\boldsymbol{\Psi}_{1}$ can be acquired by neglecting the square term $c^{2} \mathbf{n}_{1}^{2}$ as

$$
\boldsymbol{\Psi}_{1}=E\left[\psi_{1} \psi_{1}^{T}\right]=4 c^{2} \mathbf{B}_{1} E\left[\mathbf{n}_{1} \mathbf{n}_{1}^{T}\right] \mathbf{B}_{1}=4 c^{2} \mathbf{B}_{1} \mathbf{Q}_{1} \mathbf{B}_{1}
$$

where

$$
\begin{aligned}
& \mathbf{B}_{1}=\operatorname{diag}\left\{\zeta_{2}, \ldots, \zeta_{N\left(\mathbf{B}_{l}\right)}, \zeta_{1} \cos \phi, \zeta_{1}, \tilde{\zeta}_{1}, \ldots, \tilde{\zeta}_{N\left(\mathbf{S}_{g}\right)}\right\} \\
& \mathbf{Q}_{1}=\operatorname{diag}\left\{\sigma_{r_{2,1}}^{2}, \ldots, \sigma_{r_{N\left(\mathbf{B}_{l}\right), 1}}^{2}, \sigma_{r_{\theta}}^{2}, \sigma_{r_{\phi}}^{2}, \sigma_{\tilde{r}_{1}}^{2}, \ldots, \sigma_{\tilde{r}_{N\left(\mathbf{S}_{g}\right)}}^{2}\right\} .
\end{aligned}
$$

The weighted LS formulation can therefore be performed to acquire the approximate values of the variables within $\boldsymbol{x}_{H}^{(1)}$. By assigning $\ell=1$ in (12), it can be obtained that $r_{1}=\left(x^{2}+\right.$ $\left.y^{2}+z^{2}-2 x_{1} x-2 y_{1} y-2 z_{1} z+\kappa_{1}\right)^{1 / 2}$. To provide hybrid location estimation for both the TOA and TDOA/AOA measurements, the relationship between (12) and (13) should be established (i.e., between $\beta$ and $r_{1}$ ). With coordinate transformation, it is feasible to assume that the home BS is located at the origin, i.e., $\left(x_{1}, y_{1}, z_{1}\right)=(0,0,0)$. The variable $r_{1}^{2}$ becomes $r_{1}^{2}=\left(x^{2}+y^{2}+z^{2}\right)$ within (13), which can consequently result in the relationship of $\beta=r_{1}^{2}-b^{2}$ based on (12). Therefore, the relationship could be applied, and the elements within the second step of the HLS estimator formulation (i.e., $\mathbf{M}_{2} \boldsymbol{x}_{H}^{(2)}=$ $\mathbf{J}_{2}$ ) can be obtained as

$$
\mathbf{M}_{2}=\left[\begin{array}{lllll}
1 & 0 & 0 & 0 & 0 \\
0 & 1 & 0 & 0 & 0 \\
0 & 0 & 1 & 0 & 0 \\
0 & 0 & 0 & 1 & 0 \\
0 & 0 & 0 & -1 & 1 \\
0 & 0 & 0 & 0 & 1
\end{array}\right] \quad \mathbf{J}_{2}=\left[\begin{array}{c}
\left(x^{(1)}\right)^{2} \\
\left(y^{(1)}\right)^{2} \\
\left(z^{(1)}\right)^{2} \\
\left(b^{(1)}\right)^{2} \\
\beta^{(1)} \\
\left(r_{1}^{(1)}\right)^{2}
\end{array}\right]
$$

with $\boldsymbol{x}_{H}^{(2)}=\left[\begin{array}{lllll}\left(x^{(2)}\right)^{2} & \left(y^{(2)}\right)^{2} & \left(z^{(2)}\right)^{2} & \left(b^{(2)}\right)^{2} & \left(r_{1}^{(2)}\right)^{2}\end{array}\right]^{T}$, which can also be solved by the weighted LS formulation with the weighting matrix $\boldsymbol{\Psi}_{2}$ of the second step as

$$
\boldsymbol{\Psi}_{2}=4 \mathbf{B}_{2} \operatorname{cov}\left(\boldsymbol{x}_{H}^{(1)}\right) \mathbf{B}_{2}=4 \mathbf{B}_{2}\left(\mathbf{M}_{1}^{T} \boldsymbol{\Psi}_{1}^{-1} \mathbf{M}_{1}\right)^{-1} \mathbf{B}_{2}
$$

where $\mathbf{B}_{2}=\operatorname{diag}\left\{x^{(1)}, y^{(1)}, z^{(1)}, b^{(1)}, 1 / 2, r_{1}^{(1)}\right\}$. With the relationship of $\beta=r_{1}^{2}-b^{2}$, it can be observed that the variable $\beta$ is removed from $\boldsymbol{x}_{H}^{(1)}$ to form the reduced dimension vector of $\boldsymbol{x}_{H}^{(2)}$. Furthermore, the third step of the HLS estimator is, again, to incorporate the relationship with $\left(r_{1}^{(2)}\right)^{2}=\left(x^{(3)}\right)^{2}+$ $\left(y^{(3)}\right)^{2}+\left(z^{(3)}\right)^{2}$ into the matrix formulation (i.e., $\mathbf{M}_{3} \boldsymbol{x}_{H}^{(3)}=$ $\left.\mathbf{J}_{3}\right)$ as

$$
\mathbf{M}_{3}=\left[\begin{array}{llll}
1 & 0 & 0 & 0 \\
0 & 1 & 0 & 0 \\
0 & 0 & 1 & 0 \\
0 & 0 & 0 & 1 \\
1 & 1 & 1 & 0
\end{array}\right] \quad \mathbf{J}_{3}=\left[\begin{array}{c}
\left(x^{(2)}\right)^{2} \\
\left(y^{(2)}\right)^{2} \\
\left(z^{(2)}\right)^{2} \\
\left(b^{(2)}\right)^{2} \\
\left(r_{1}^{(2)}\right)^{2}
\end{array}\right]
$$

with $\boldsymbol{x}_{H}^{(3)}=\left[\begin{array}{llll}\left(x^{(3)}\right)^{2} & \left(y^{(3)}\right)^{2} & \left(z^{(3)}\right)^{2} & \left(b^{(3)}\right)^{2}\end{array}\right]^{T}$. The associated weighting matrix $\Psi_{3}$ of the third step becomes

$$
\boldsymbol{\Psi}_{3}=\mathbf{B}_{3} \operatorname{cov}\left(\boldsymbol{x}_{H}^{(2)}\right) \mathbf{B}_{3}=\mathbf{B}_{3}\left(\mathbf{M}_{2}^{T} \boldsymbol{\Psi}_{2}^{-1} \mathbf{M}_{2}\right)^{-1} \mathbf{B}_{3}
$$

where $\mathbf{B}_{3}$ is an identical matrix, which indicates that the third step of the HLS estimator is exploited as the tuning process based on the second step's covariance matrix $\operatorname{cov}\left(\boldsymbol{x}_{H}^{(2)}\right)$. The final estimated MSs position, using the proposed HLS estimator, can be obtained as $\boldsymbol{x}_{H}=\mathbf{T} \cdot\left(\boldsymbol{x}_{H}^{(3)}\right)^{1 / 2}$, where $\mathbf{T}=$ $\operatorname{diag}(1,1,1,0)$. The effectiveness of the HLS estimator will be evaluated in the simulations.

\section{Performance Evaluation}

The performance of the proposed hybrid architectures is evaluated through simulations and is described in the following sections. Section V-A addresses the assessments on the proposed schemes according to time complexity. The noise models that were utilized in the simulations are shown in Section V-B. Section V-C describes the validation of the hybrid architectures and the associated HLS estimator under the circumstance with pure measurement noises. Section V-D illustrates the performance evaluation of the proposed schemes under the NLOS environments. The following two cases are considered: 1) different circumstances with a fixed set of available signal inputs and 2) realistic environments with either a stationary or a moving MS.

\section{A. Assessments on the Proposed Architectures and Schemes}

To illustrate the feasibility of the proposed architectures, the assessments for the corresponding approaches are discussed in this section in view of time complexity. Note that both the FH and UH architectures can be implemented as either the mobile-assisted or the mobile-based system. In the mobilebased system, except for the AOA, information needs to be delivered from the BS to the MS, and all the required information for location estimation and tracking is calculated and processed within the MS. On the other hand, considerable message transmissions between the MS and the BS should be conducted by adopting the mobile-assisted system to allow the BS to obtain the MSs position. Therefore, provided that the MS is possessed with sufficient processing capability, the mobilebased system is considered to be a favorable architecture for location estimation of the MS. However, due to the huge processing load within the MS by adopting the mobile-based system, efficient power management should be considered to reduce power consumption within the MS. 
TABLE I

Number OF REQUiRED COMPUTATIONS OF THE FH ARCHITECTURE

\begin{tabular}{|c|c|c|c|}
\hline & Value $p$ & Value $q$ & Computation costs \\
\hline Satellite-based $1^{s t}$ step & 5 & 5 & 1400 \\
\hline Satellite-based $2^{\text {nd }}$ step & 5 & 4 & 1214 \\
\hline Cell-based $1^{\text {st }}$ step & 4 & 4 & 720 \\
\hline Cell-based $2^{\text {nd }}$ step & 4 & 3 & 603 \\
\hline Total & & & 3937 \\
\hline
\end{tabular}

TABLE II

NUMber of REQUiRED COMPUTATIONS OF THE UH ARCHITECTURE

\begin{tabular}{|c|c|c|c|}
\hline & Value $p$ & Value $q$ & Computation costs \\
\hline$\overline{H L S} 1^{s t}$ step & 9 & 6 & 6426 \\
\hline HLS $2^{\text {nd }}$ step & 6 & 5 & 2141 \\
\hline HLS $3^{r d}$ step & 5 & 4 & 1214 \\
\hline Total & & & 9781 \\
\hline
\end{tabular}

Moreover, the assessment between the FH and UH architectures is discussed. Basically, two pairs of location estimators and Kalman filters are adopted for the FH scheme, whereas an integrated technique is exploited in the $\mathrm{UH}$ architecture. To illustrate the comparison between the FH and UH structures, one example is given under the situation with $N\left(\mathbf{S}_{q}\right)=5$ and $N\left(\mathbf{B}_{l}\right)=4$, which corresponds to $N\left(\mathbf{H}_{s}\right)=9$. First, the time complexity of the two-step LS method and the proposed HLS scheme can be obtained by considering both matrix multiplication and matrix inversion within the LS estimator. Note that the multiplication between an $i \times j$ matrix and an $j \times k$ matrix runs in $O(i \cdot j \cdot k)$, whereas the computation cost of matrix inversion for an $i \times i$ matrix can be approximated as $O\left(i^{3}\right)$ [36]. Provided that the number of measurement inputs is $p$ and the number of variables that will be solved is $q$, the computation cost for each LS iteration step becomes $O\left(6 p^{3}\right)+O\left(q^{3}\right)+$ $O\left(3 p^{2} q\right)+O\left(p q^{2}\right)+O(p q)$ by evaluating the complexity in (15) and (16). Therefore, the total number of computations for the FH and UH architectures can be obtained as listed in Tables I and II, respectively. On the other hand, provided that the number of measurement inputs is $u$ and the number of state vectors is $w$, the computation cost for the Kalman filter can be acquired as $O\left(u^{3}\right)+O\left(w^{3}\right)+O\left(2 u^{2} w\right)+O\left(2 u w^{2}\right)$ for each iteration [37]. Considering the case that $u=3$ and $w=9$, the computation costs for the $\mathrm{FH}$ and $\mathrm{UH}$ architectures can be obtained as 2808 and 1404, respectively, because two separate Kalman filters are required for the FH scheme. Therefore, the total computation costs from both the location estimator and the Kalman filter for the FH and $\mathrm{UH}$ architectures become 6745 and 11185 , respectively. With a smaller size of measurement input by adopting separate estimation paths, the $\mathrm{FH}$ architecture will possess less computation load compared with the $\mathrm{UH}$ structure.

Furthermore, the complexity of both FSI and SSI methods is compared within the FH architecture. One example is considered with the GPS-SSI and GPS-FSI schemes under the situation in which $N\left(\mathbf{S}_{g}\right)=7$. Compared with the GPS-SSI scheme, the GPS-FSI method additionally performs the Optimum5 algorithm to restrict the number of measurement inputs to 5. The Optimum5 function is required to search $C_{5}^{N\left(\mathbf{S}_{g}\right)}$ times for signal selection, i.e., $C_{5}^{7}=21$ times of computation. On the other hand, considering the computation cost for implementing the two-step LS method, the GPS-FSI scheme will conserve $3128-1400=1728$ times of calculation compared with the GPS-SSI approach. As a result, the FSI scheme can conserve the computation cost by reducing the number of measurement inputs, whereas the SSI approach will, in general, provide better estimation performance with its additional usage of signal sources. Performance comparisons between these proposed architectures and approaches will be conducted and validated in the following sections.

\section{B. Noise Models}

Different noise models [8], [35], [38], [39] are considered in the simulations to represent various environments, including both urban and rural. The probability distribution of the noise model $p_{\rho_{\ell}}\left(\tau_{g}\right)$ for the set of satellites is selected as

$$
p_{\rho_{\ell}}\left(\tau_{g}\right) \sim \mathcal{N}\left(0, \sigma_{\rho_{\ell}}^{2}\left(\tau_{g}\right)\right)
$$

where the variance is defined as $\sigma_{\rho_{\ell}}\left(\tau_{g}\right)=\left[a+b^{2}\right.$. $\left.10^{-\tau_{g} / 10}\right]^{1 / 2}$. The parameters $a=10 \mathrm{~m}^{2}$ and $b=150 \mathrm{~m}^{2}$ are determined by user equipment. The SNR value $\tau_{g}$, which is adopted from [38] as an input to a variance model for the GPS pseudorange measurement, is a uniform distributed random variable. To model good signal quality (i.e., $\mathbf{S}_{g}$ ), $\tau_{g}$ is selected in the intervals of $(20,30) \mathrm{dB}$ and $(30,45) \mathrm{dB}$ for the urban and rural environments, respectively. On the other hand, $\tau_{g} \sim U(10,20) \mathrm{dB}$ is chosen to represent the situations with bad signal quality, i.e., $\mathbf{S}_{b}$. The value of the clock bias $b$ is selected as $1.7 \times 10^{-6}$ s (i.e., $500 \mathrm{~m}$ ) for good signal quality, whereas twice the value is assigned for the satellite with bad signal quality. In the cellular-based network, an exponential distribution is assumed for the NLOS model with the distribution of $p_{n_{n l, i}}(v)$ as

$$
p_{n_{n l, i}}(v)= \begin{cases}\frac{1}{v_{i}} e^{\frac{-v}{v_{i}}}, & v>0 \\ 0, & v \leq 0\end{cases}
$$

where $v_{i}=c \cdot \tau_{i}=c \cdot \tau_{m} \zeta_{i}^{\varepsilon} \omega$ is the root mean square (RMS) delay spread between the $i$ th BS to the MS, and $\tau_{m}$ is the median value of $\tau_{i}$, whose value is selected as $0.4 \mu \mathrm{s}$ and $0.1 \mu \mathrm{s}$ for the urban and rural environments, respectively. $\varepsilon$ is the path loss exponent, which is assumed to be 0.5. The shadow-fading factor $\omega$ is a log-normal random variable with zero mean and standard deviation $\sigma_{\omega}$, which was chosen as $4 \mathrm{~dB}$ in the simulations. Moreover, the measurement noises for TOA [i.e., $n_{\ell}$ in (2)] and TDOA [i.e., $n_{i}$ and $n_{j}$ in (4)] are considered Gaussian distributed as $\mathcal{N} \sim\left(0, \sigma_{n_{\xi}}^{2}\right)$, with $\sigma_{n_{\xi}}=$ $10 \mathrm{~m}$ for $\xi=\ell, i$, and $j$. Similarly, the noise models for the AOA measurements [i.e., $n_{\theta}$ and $n_{\phi}$ in (5) and (6)] are both assumed to be Gaussian distributed, with $\mathcal{N} \sim\left(0, \tau_{a}^{2}\right)$, where $\tau_{a}$ is chosen as $10^{\circ}$ and $5^{\circ}$, respectively, for the urban and rural environments. 


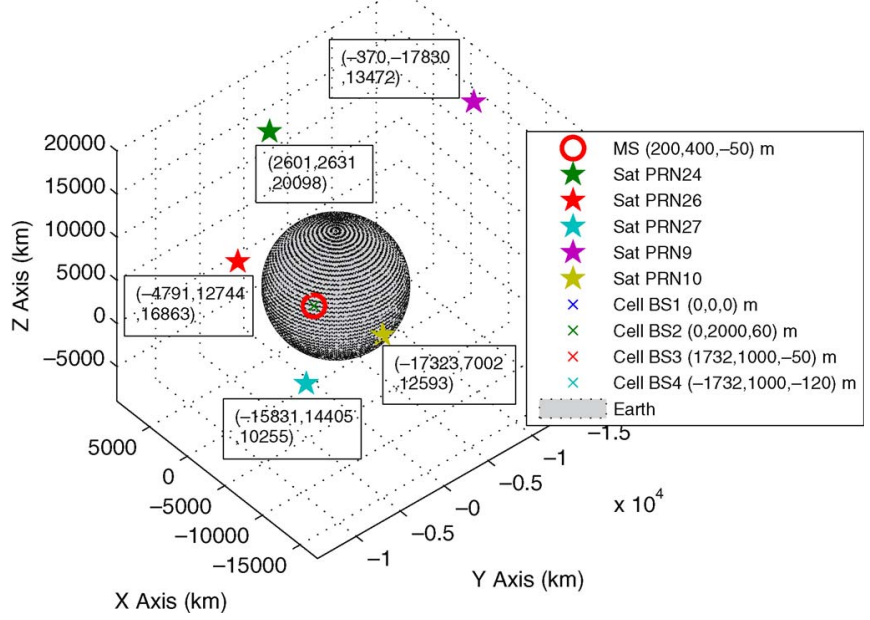

Fig. 4. Simulation parameters for Sections V-C, D1, and D2. Graphical representation for the coordinates of satellites that were marked in the textbox (in kilometers), cell BSs (in meters), and MS (in meters).
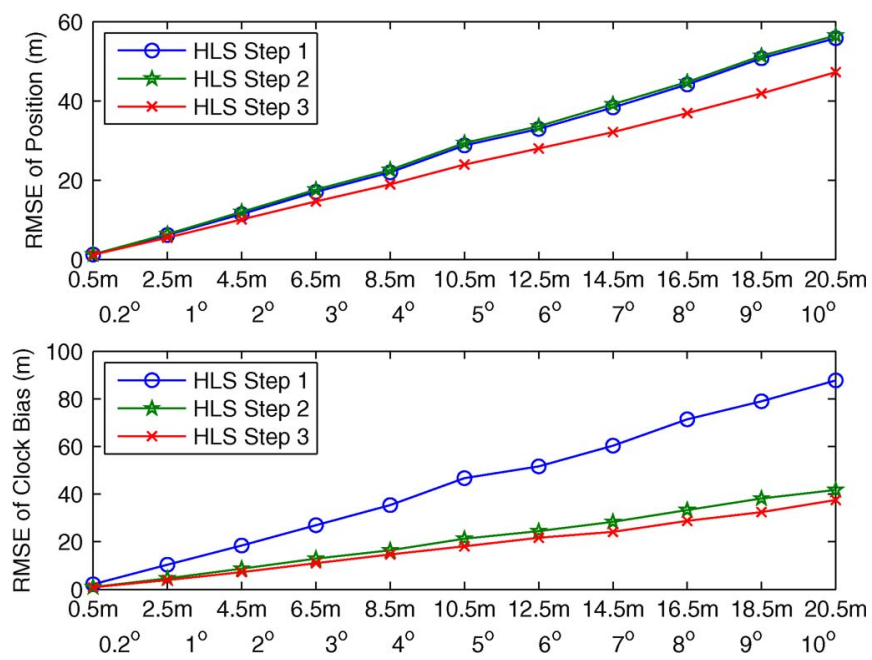

Fig. 5. Performance comparison between each step of the HLS estimator under pure measurement noises. (Top) RMSE of position and (bottom) RMSE of clock bias versus the standard deviation of measurement noises $\left(\sigma_{n_{\xi}}, \sigma_{n_{\theta}}\right.$, and $\left.\sigma_{n_{\phi}}\right)$.

\section{Validation of the Proposed Algorithms With Pure Measurement Noises}

The HLS estimator, as proposed in Section IV-B, is validated under the environment with pure measurement noises, i.e., only $n_{\xi}$ (for $\xi=\ell, i$, and $j$ ), $n_{\theta}$, and $n_{\phi}$ are considered. Fig. 4 shows the simulation parameters for performance validation. Fig. 5 illustrates the comparison of the RMS error (RMSE) for the MSs estimated position (upper plot) and the clock bias (lower plot) between the three steps of the HLS estimator under different standard deviations of the measurement noises $\sigma_{n_{\xi}}$, $\sigma_{n_{\theta}}$, and $\sigma_{n_{\phi}}$. Note that the RMSE is computed as RMSE = $\left[\sum_{i=1}^{N_{r}}\|\mathbf{x}-\hat{\boldsymbol{x}}(i)\|^{2} / N_{r}\right]^{1 / 2}$, where $N_{r}=10000$ indicates the number of simulation runs. In addition, $\mathbf{x}$ represents the true position of the MS, whereas $\hat{\boldsymbol{x}}(i)$ denotes the MSs estimated position in the upper plot of Fig. 5. On the other hand, $x$ indicates the clock bias (chosen as $50000 \mathrm{~m}$ in this case), and

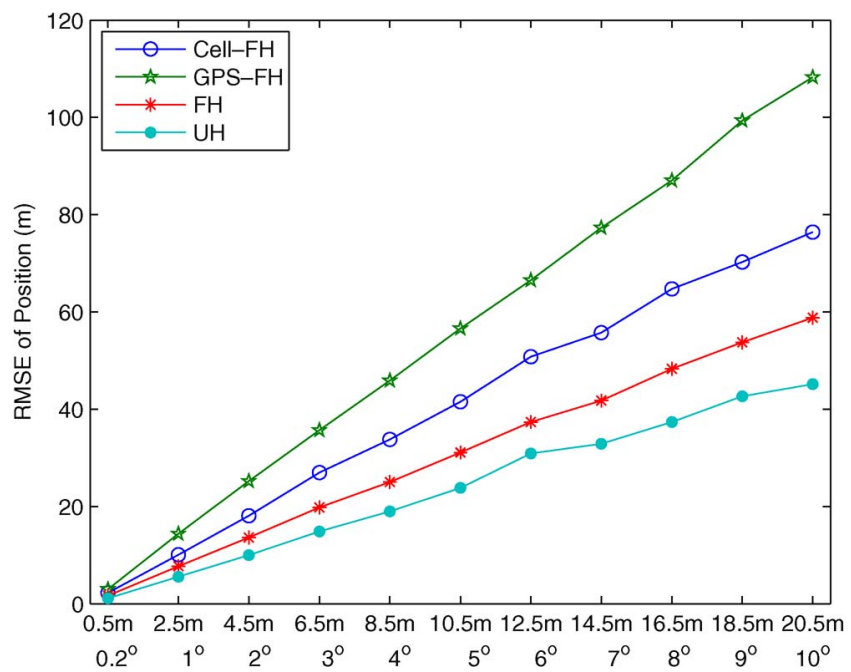

Fig. 6. Performance comparison between the location-estimation schemes under pure measurement noises. RMSE of position versus standard deviation of measurement noises $\left(\sigma_{n_{\xi}}, \sigma_{n_{\theta}}\right.$, and $\left.\sigma_{n_{\phi}}\right)$.

$\hat{\boldsymbol{x}}(i)$ corresponds to the estimated clock bias in the lower plot of Fig. 5. It can be observed that the RMSEs of the MSs estimated position are significantly decreased from Steps 2 to 3 , whereas the RMSEs of the clock bias are considerably reduced from Steps 1 to 2 . The reason can be attributed to the implicit different weights that are imposed by the distinctive steps within the proposed HLS estimator. The mechanism within the second step of the HLS estimator removes the clock bias $b$ by adopting the relationship between the intermediate variables $\beta$ and $r_{1}$, i.e., $\beta=r_{1}^{2}-b^{2}$. On the other hand, the association between $r_{1}$ and the MSs position is constructed within the third step of the HLS scheme, i.e., $r_{1}=x^{2}+y^{2}+z^{2}$. Consequently, the results that were obtained based on Fig. 5 validate the effectiveness of the proposed HLS estimator for location estimation of the MS.

Fig. 6 shows the performance comparison between the proposed FH $\left(\hat{\boldsymbol{x}}_{f}\right)$ and UH $\left(\hat{\boldsymbol{x}}_{H}\right)$ schemes, which were associated with the Cell-FH $\left(\hat{\boldsymbol{x}}_{C}\right)$ and the GPS-FH $\left(\hat{\boldsymbol{x}}_{G}\right)$ methods. Note that the Cell-FH and GPS-FH schemes are denoted as the separate channels within the FH architecture without the implementation of the data fusion. For example, the Cell-FH scheme includes the building blocks of the CSS, the two-step LS method, and the Kalman filter, as shown in the left diagram in Fig. 2. Both schemes are utilized in this figure and the remaining sections to illustrate the performance for location estimation, whereas only a single type of channels (either the cellular- or the satellite-based network) is exploited. In addition, note that both the FSI and SSI schemes are considered the same, because a minimum number of required signal inputs are adopted in this case. Moreover, online adaption for the datafusion mechanism as in (1) is performed in the simulations. The signal means and variances, i.e., $\left(\underline{\hat{x}}_{G}, \boldsymbol{\sigma}_{G}\right)$ and $\left(\underline{\hat{x}}_{C}, \boldsymbol{\sigma}_{C}\right)$, are obtained based on the acquisition of the previous ten estimated data points of $\hat{\boldsymbol{x}}_{G}$ and $\hat{\boldsymbol{x}}_{C}$, respectively. It can be observed that the proposed $\mathrm{UH}$ and $\mathrm{FH}$ architectures outperform the other two schemes under various noise environments. The effectiveness of the data fusion within the FH scheme is validated. With the incorporation of the HLS estimator, the UH scheme can provide 
TABLE III

NLOS BSS IDENTIFICATION RATE FOR THE FH SCHEME UNDER AN URBAN ENVIRONMENT

\begin{tabular}{ccccc}
\hline \multirow{2}{*}{$\begin{array}{c}\text { Number of NLOS } \\
\text { BSs: } N\left(\mathbf{B}_{n}\right)\end{array}$} & $\begin{array}{c}\text { Number of NLOS BS } \\
\text { Correctly Detected }\end{array}$ & \multicolumn{2}{c}{$N\left(\mathbf{B}_{t}\right)\left[N\left(\mathbf{S}_{t}\right)\right]$} \\
\cline { 3 - 5 } 1BS & 1BS & 0.778 & 0.852 & 0.898 \\
\hline 2BSs & 2BSs & 0.443 & 0.678 & 0.839 \\
& 1BS & 0.084 & 0.054 & 0.003 \\
\hline 3BSs & 3BSs & & 0.459 & 0.645 \\
& 2BSs & & 0.115 & 0.051 \\
& 1BS & & 0.016 & 0.137 \\
\hline
\end{tabular}

TABLE IV

NLOS BSs IDENTIFICATION RATE FOR THE UH SCHEME UNDER AN URBAN ENVIRONMENT

\begin{tabular}{ccccc}
\hline \multirow{2}{*}{$\begin{array}{c}\text { Number of NLOS } \\
\text { BSs: } N\left(\mathbf{B}_{n}\right)\end{array}$} & Number of NLOS BS & \multicolumn{3}{c}{ Number of BS } \\
\cline { 3 - 5 } & Correctly Detected & $4[5]$ & $5[5]$ & $6[5]$ \\
\hline 1BS & 1BS & 0.862 & 0.907 & 0.945 \\
\hline 2BSs & 2BSs & 0.732 & 0.800 & 0.890 \\
& 1BS & 0.246 & 0.050 & 0.006 \\
\hline 3BSs & 3BSs & & 0.634 & 0.728 \\
& 2BSs & & 0.321 & 0.050 \\
& 1BS & & 0.034 & 0.049 \\
\hline
\end{tabular}

better performance compared with the FH method, particularly with a larger standard deviation of the measurement noises.

\section{Simulation Results Under NLOS Environments}

1) Identification of BSs With NLOS Errors: As explained in Section III-B, a residual test is performed for the identification of the BSs with NLOS errors. Tables III and IV show the identification rates (i.e., the rates to correctly detect the BS(s) with NLOS error) for both the FH and UH schemes under an urban environment. The test threshold $\gamma_{t h}=0.9$ is chosen to achieve a low false-alarm rate that is associated with 10000 trials of the residual test. For instance, the identification rate to correctly obtain two NLOS BSs under the situation of two NLOS BSs (with $N\left(\mathbf{B}_{t}\right)\left[N\left(\mathbf{S}_{t}\right)\right]=5[5]$ ) is 0.678 for the $\mathrm{FH}$ scheme and 0.800 for the UH method. It can be observed in Tables III and IV that the UH scheme can provide a higher identification rate compared with that from the FH method. The reason can be attributed to the better initial location estimate of the MS $\left(\hat{\boldsymbol{x}}_{C}^{o}\right)$ that was acquired from the UH structure.

2) Environments With a Fixed Set of Available Signal Inputs: In this scenario, a fixed set of available signal inputs are considered, i.e., five satellites $\left(N\left(\mathbf{S}_{t}\right)=5\right)$ and four BSs $\left(N\left(\mathbf{B}_{t}\right)=4\right)$ are accessible. The signal inputs that are adopted within the FH scheme is considered fixed, whereas the signalselection scheme within the UH scheme (i.e., the HSS-SSI in Algorithm 3) chooses signals with better quality, which may result in a smaller number of signal sources (i.e., in case that $N\left(\mathbf{S}_{g}\right)<5$ or $N\left(\mathbf{B}_{l}\right)<4$ ). The simulation parameters are shown in Fig. 4. Fig. 7 illustrates the performance comparison between the four schemes under the urban (left plot) and rural (right plot) environments. Note that the position errors $\Delta x$ are computed as $\Delta x=\left[\sum_{i=1}^{N_{r}}\|\boldsymbol{x}-\hat{\boldsymbol{x}}(i)\|\right] / N_{r}$, where $N_{r}=1000$ indicates the number of simulation runs. Different
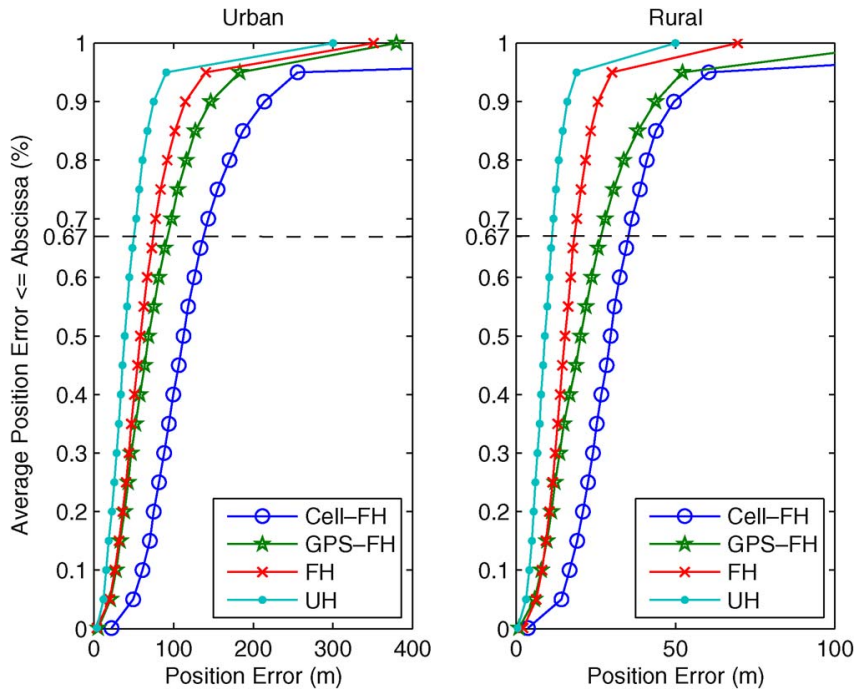

Fig. 7. (Left) Performance comparison under an urban environment with 50\% of NLOS BSs and $20 \%$ of bad satellites. (Right) Performance comparison under a rural environment with $50 \%$ of NLOS BSs and $0 \%$ of bad satellites.

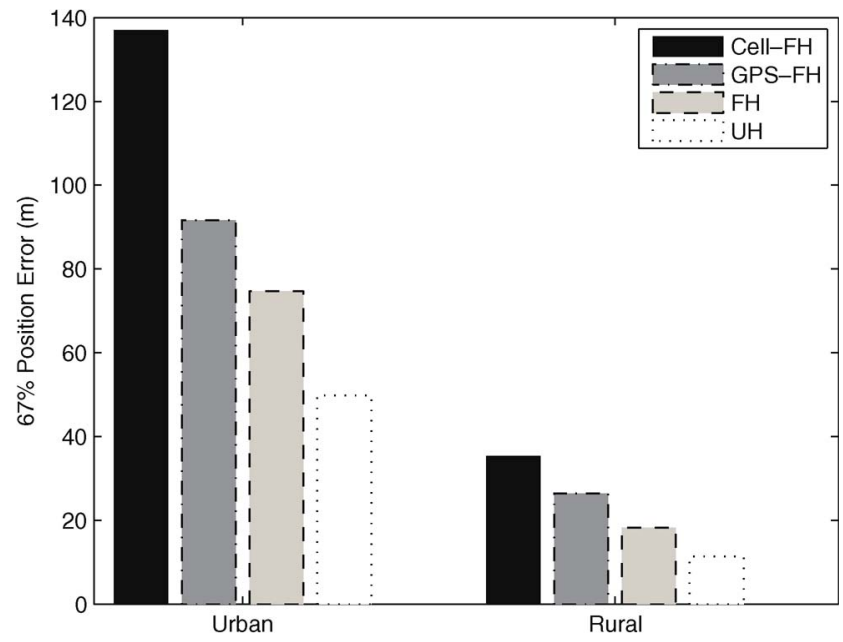

Fig. 8. Performance comparison with $67 \%$ position error under two different environments. The bars from left to right indicate the Cell-FH, GPS-FH, FH, and UH schemes.

signal qualities of the satellites and the NLOS errors in the cellular signals are considered, as shown in Fig. 7. In both environments, it can be observed that the proposed UH scheme can provide better performance compared with the other three methods under different percentages of position errors. It can be observed that the Cell-FH scheme is shown perform worse compared with the GPS-FH method under the urban and rural environments, as shown in Fig. 7. Fig. 8 shows the performance comparison of these four schemes within the two environments under $67 \%$ of position error. Smaller position errors are obtained using the proposed UH scheme.

3) Realistic Environments With a Stationary MS: This scenario demonstrates how the proposed algorithms work in realistic situations, whereas an MS is stationary at a specific location (i.e., in an urban area) for a certain time interval. Fig. 9 shows the total available satellites $\left(N\left(\mathbf{S}_{t}\right)\right)$ and BSs $\left(N\left(\mathbf{B}_{t}\right)\right)$ that are considered every hour between 11:00 and 18:00 on 


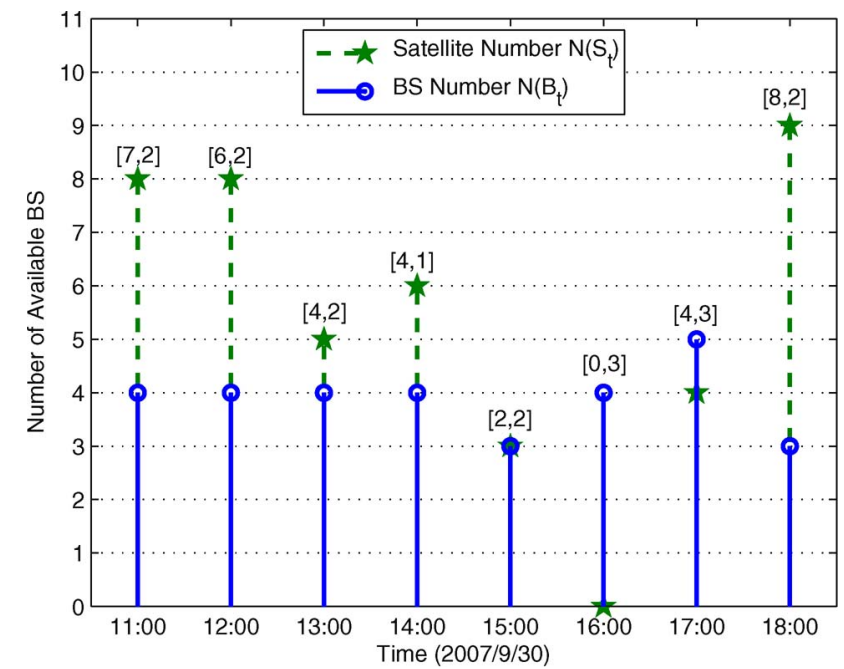

Fig. 9. Number of available satellites and BSs at different time instants (for a stationary MS). The set $\left[N\left(\mathbf{S}_{g}\right), N\left(\mathbf{B}_{l}\right)\right]$ on the graph indicates the numbers of satellites with good signal quality and BSs with LOS measurement.

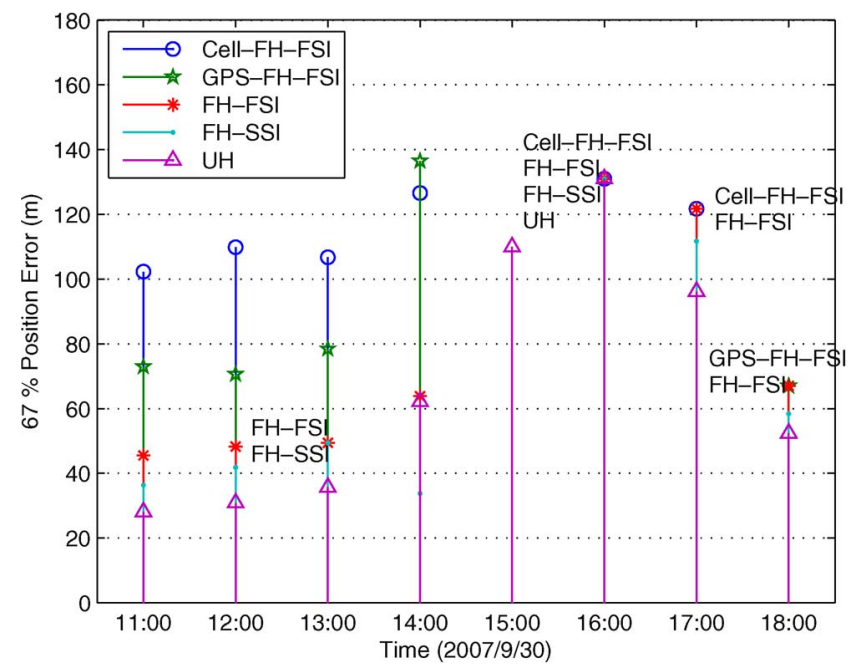

Fig. 10. Performance comparison under $67 \%$ of position error at different time instants. At 13:00, the FH-FSI and FH-SSI schemes achieve the same performance. At 16:00, the Cell-FH-FSI, FH-FSI, FH-SSI, and UH schemes achieve the same performance. At 17:00, the Cell-FH-FSI and FH-FSI schemes achieve the same performance. At 18:00, the GPS-FH-FSI and FH-FSI schemes achieve the same performance.

September 30, 2007 at (N 24.47, E 120.59, $91 \mathrm{~m}$ ) in Hsinchu, Taiwan. The coordinates of the satellites are collected from the Satscape software [40], which calculates satellite positions using NORAD SGP4 orbital models. The sets of satellites with good signal quality and the BSs with LOS measurements (i.e., $\left.\left[N\left(\mathbf{S}_{g}\right), N\left(\mathbf{B}_{l}\right)\right]\right)$ are denoted on the plot. Note that incorrect identification for both types of signal sources are also considered in the simulations.

By adopting the scenario as in Fig. 9, the performance comparison between the four different schemes can be obtained as shown in Fig. 10 (under $67 \%$ of position error). In general, the proposed UH algorithm outperforms the other four schemes in most cases. At time instant 14:00, the large estimation error (i.e., $125 \mathrm{~m}$ as in Fig. 10) that was acquired from the Cell-FH-FSI scheme can be attributed to the weak

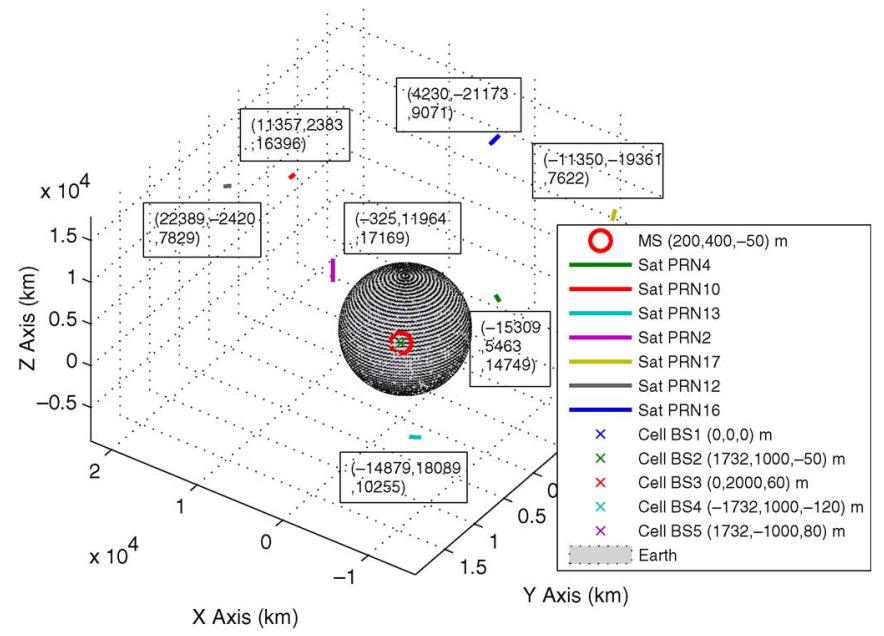

Fig. 11. Simulation parameters for Section V-D4. Graphical representation for the coordinates of moving satellites [the solid line refers to satellites trajectory during $100 \mathrm{~s}$, whereas the textbox refers to the coordinates of the satellite at time instant 13:30 (in kilometers)], cell BSs (in meters), and MS (in meters).

NLOS identification, as shown in Fig. 9, where $N\left(\mathbf{B}_{t}\right)=4$, and $N\left(\mathbf{B}_{l}\right)=1$. The fusion algorithm within the FH-SSI scheme can overcome excessive NLOS errors, which results in better location estimation compared with the other methods. At 15:00, where $\left[N\left(\mathbf{S}_{t}\right), N\left(\mathbf{B}_{t}\right)\right]=[3,3]$, all the four schemes (i.e., the GPS-FH-FSI, Cell-FH-FSI, FH-FSI, and FH-SSI methods) fail to perform location estimation for the MS due to the deficiency of signal sources. However, the UH scheme can still effectively provide consistent location estimation with a position error that is equal to $108 \mathrm{~m}$, as shown in Fig. 10 . At time instant 16:00, where the number of available satellites is zero, both the $\mathrm{FH}$ and $\mathrm{UH}$ schemes are transformed into the Cell-FH-FSI method, which utilizes the two-step LS method for location estimation. It can be observed that the same estimation error (i.e., around $130 \mathrm{~m}$ ) is obtained, as shown in Fig. 10. On the other hand, the GPS-FH-FSI scheme apparently cannot conduct location estimation for the MS. At time instant 17:00, where the number of available satellites is four, the GPS-FH-FSI scheme cannot provide location estimation. The FH-FSI scheme is transformed into the Cell-FH-FSI method, whereas the FH-SSI still adopts signal sources from both sides to achieve a lower estimation error that is equal to $111 \mathrm{~m}$. At 18:00, with $\left[N\left(\mathbf{S}_{g}\right), N\left(\mathbf{B}_{l}\right)\right]=[8,2]$, the $\mathrm{FH}$ algorithm will perform similar to the GPS-FH-FSI method due to the insufficient signals within the cell-based network. However, the UH algorithm can still provide feasible location estimation (around $15 \mathrm{~m}$ less in position error) compared with the FH-FSI scheme, even with insufficient signal inputs.

4) Realistic Environments With a Moving MS: In this scenario, the proposed schemes are compared under the environment with a moving MS, where an urban area with $50 \%$ of NLOS BSs condition is considered. Note that the trajectories of satellites that were adopted from the Satscape software [40] are approximated with the NORAD SGP4 model. The trajectories start from 13:30 on September 30, 2007 for a duration of $100 \mathrm{~s}$. The simulation parameters and scenarios are shown in Fig. 11 for the starting coordinates and the trajectories of 


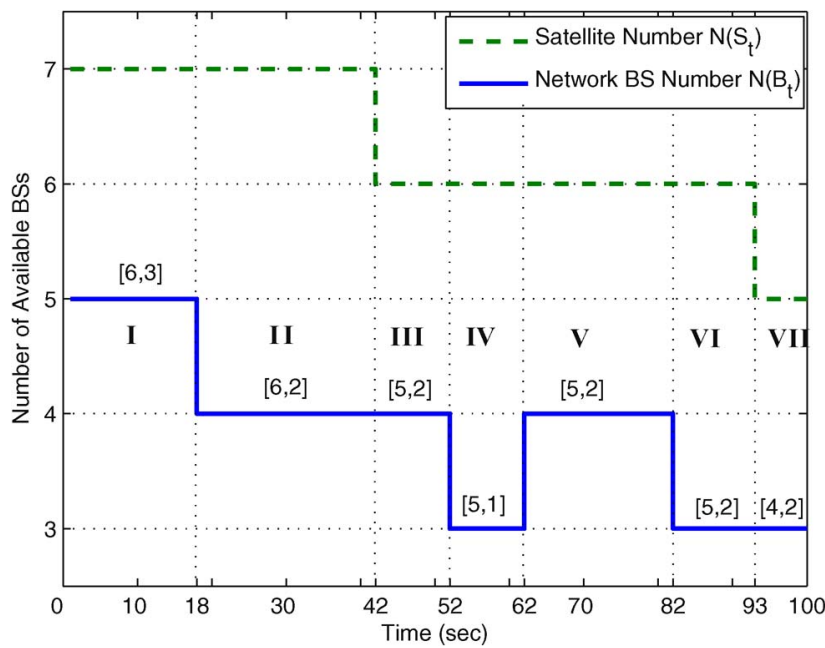

Fig. 12. Number of available satellites and BSs at different time instants (for the moving MS). The set $\left[N\left(\mathbf{S}_{g}\right), N\left(\mathbf{B}_{l}\right)\right]$ on the graph indicates the numbers of satellites with good signal quality and BSs with LOS measurement.

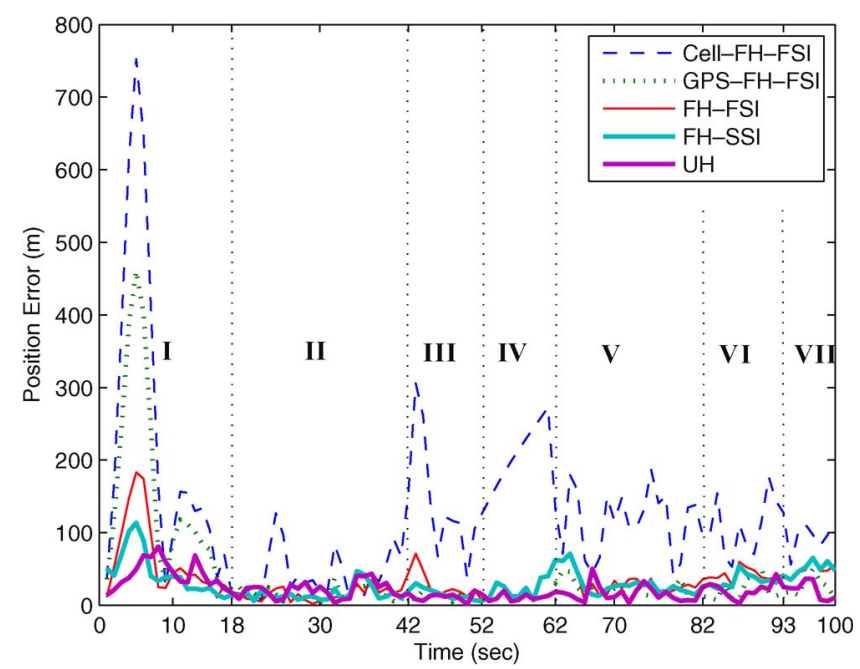

Fig. 13. Performance comparison for location estimation and tracking: Position error versus time (the region indexes correspond to the indexes as in Fig. 12).

moving satellites during this period. Moreover, the trajectory of the MS is illustrated in Fig. 14(a). The total available satellites $N\left(\mathbf{S}_{t}\right)$ and BSs $N\left(\mathbf{B}_{t}\right)$ are illustrated in Fig. 12, with the sets of $\left[N\left(\mathbf{S}_{g}\right), N\left(\mathbf{B}_{l}\right)\right]$ indicated on the plot.

Fig. 13 shows the position errors between the four schemes under the topology, as shown in Fig. 12 (with a time duration of $100 \mathrm{~s}$ ). It can be observed that the proposed UH algorithm can provide better performance for location estimation and tracking compared with the other four methods. Note that the Kalman filter within all the four algorithms becomes effective for MSs location tracking in this case. In Region 1, excessive position errors are observed in all the five schemes due to the transient state of their Kalman filters. However, the error-convergent rates are tolerable due to the sufficient signal sources that are available for location estimation. On the other hand, signal deficiency happens within the cellular channel in Regions 4, 6, and 7. The GPS-FH-FSI, FH-FSI, FH-SSI, and UH algorithms can still conduct persistent location estimation using the sources from the satellite signals. The Cell-FH-FSI method can only perform linear prediction within its Kalman filtering technique, which results in comparably worse performance for location tracking.

Fig. 14(a)-(c) show the trajectory tracking for the MSs position, velocity, and acceleration between the two selective sets of signal-selection schemes, i.e., the FH-SSI and UH approaches. The MS is considered to be tracked in a 2-D $x y$ plane setting. It is shown that the UH scheme outperforms the FH-SSI method for location tracking of the MS. The performance problem within the FH-SSI scheme primarily comes from the variations on the number of available signal inputs, whereas the $\mathrm{UH}$ algorithm can provide satisfactory performance for location estimation by adjusting itself under a diverse range of available signal sources.

\section{CONCLUSION}

In this paper, two different hybrid architectures for location estimation and tracking of MSs have been proposed. By combining the satellite- and the network-based systems, the proposed FH-FSI architecture can provide adequate precision for location estimation within existing infrastructure. Moreover, the FH-SSI further improves the estimation accuracy, particularly under NLOS environments. On the other hand, the UH architecture can achieve higher reliability for location estimation and tracking with its flexible architecture in most of the cases, even with deficient signal sources from heterogeneous networks. It has been shown in the simulation results that the proposed hybrid schemes can provide consistent location estimation accuracy under different environments.

\section{APPENDIX}

The formulation of the Kalman filter in Section II-A1 is explained as follows. The measurement and state equations for the Kalman filter can be represented as

$$
\begin{aligned}
& \boldsymbol{z}_{k}=\mathbf{E} \hat{\boldsymbol{s}}_{k}+\boldsymbol{m}_{k} \\
& \hat{\boldsymbol{s}}_{k}=\mathbf{F} \hat{\boldsymbol{s}}_{k-1}+\boldsymbol{p}_{k}
\end{aligned}
$$

where $\hat{\boldsymbol{s}}_{k}$ represents the output, and $\boldsymbol{z}_{k}$ denotes the measurement input of the Kalman filter. The variables $\boldsymbol{m}_{k}$ and $\boldsymbol{p}_{k}$ denote the measurement and the process noises that are associated with the covariance matrices $\mathbf{R}$ and $\mathbf{Q}$ within the Kalman filtering formulation. For the FH architecture (as shown in the left diagrams in Figs. 2 and 3), the output of the Kalman filter corresponds to either $\hat{\boldsymbol{s}}_{k}=\hat{\boldsymbol{x}}_{C}$ or $\hat{\boldsymbol{s}}_{k}=\hat{\boldsymbol{x}}_{G}$, whereas the measurement input $\boldsymbol{z}_{k}=\boldsymbol{x}_{C}$ or $\boldsymbol{z}_{k}=\boldsymbol{x}_{G}$ is obtained from the outputs of the two-step LS estimator at the $k$ th time step. On the other hand, for the UH architecture (as shown in the right diagrams in Figs. 2 and 3), the output of the Kalman filter corresponds to $\hat{\boldsymbol{s}}_{k}=\left[\begin{array}{lll}\hat{\boldsymbol{x}}_{k} & \hat{\boldsymbol{v}}_{k} & \hat{\boldsymbol{a}}_{k}\end{array}\right]^{T}$, whereas the measurement input $\boldsymbol{z}_{k}=\boldsymbol{x}_{H}$ can be acquired from the proposed HLS 

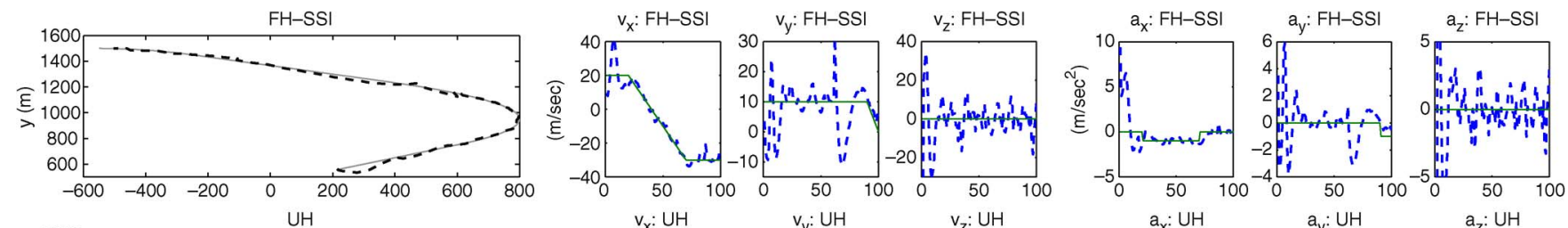

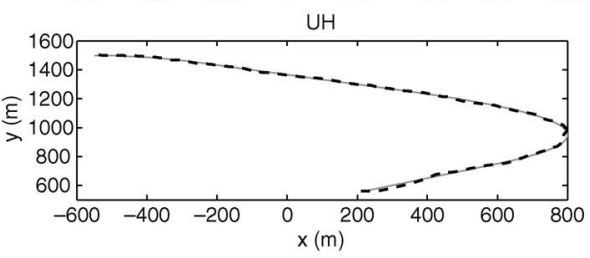

(a)

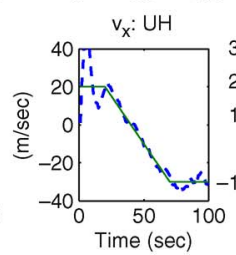

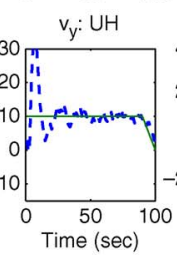

(b)
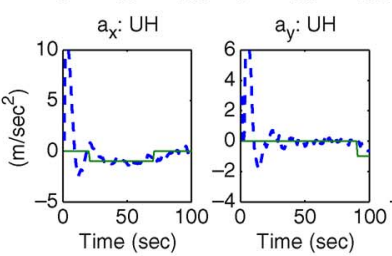

(c)

Fig. 14. Performance comparison of MS tracking using (top) the FH(SSI and (bottom) UH schemes (solid lines refer to true value, whereas dotted lines refer to estimated trajectories). (a) Trajectory. (b) Velocity. (c) Acceleration.

estimator. Furthermore, the matrix $\mathbf{E}$ and the state transition matrix $\mathbf{F}$ can be obtained as

$$
\begin{aligned}
\mathbf{E} & =\left[\begin{array}{ccccccccc}
1 & 0 & 0 & 0 & 0 & 0 & 0 & 0 & 0 \\
0 & 1 & 0 & 0 & 0 & 0 & 0 & 0 & 0 \\
0 & 0 & 1 & 0 & 0 & 0 & 0 & 0 & 0
\end{array}\right] \\
\mathbf{F} & =\left[\begin{array}{ccccccccc}
1 & 0 & 0 & \Delta t & 0 & 0 & \frac{1}{2} \Delta t^{2} & 0 & 0 \\
0 & 1 & 0 & 0 & \Delta t & 0 & 0 & \frac{1}{2} \Delta t^{2} & 0 \\
0 & 0 & 1 & 0 & 0 & \Delta t & 0 & 0 & \frac{1}{2} \Delta t^{2} \\
0 & 0 & 0 & 1 & 0 & 0 & \Delta t & 0 & 0 \\
0 & 0 & 0 & 0 & 1 & 0 & 0 & \Delta t & 0 \\
0 & 0 & 0 & 0 & 0 & 1 & 0 & 0 & \Delta t \\
0 & 0 & 0 & 0 & 0 & 0 & 1 & 0 & 0 \\
0 & 0 & 0 & 0 & 0 & 0 & 0 & 1 & 0 \\
0 & 0 & 0 & 0 & 0 & 0 & 0 & 0 & 1
\end{array}\right]
\end{aligned}
$$

where $\Delta t$ denotes the sample time interval. Based on both the prediction and updating processes within the Kalman filtering formulation, the trajectory of the MS can be predicted and traced with reasonable estimation accuracy.

\section{REFERENCES}

[1] Enhanced 911: Wireless Services, Washington, DC: Fed. Commun. Comm. [Online]. Available: http://www.fcc.gov/911/enhanced/

[2] S. Feng and C. L. Law, "Assisted GPS and its impact on navigation in intelligent transportation systems," in Proc. IEEE 5th Int. Conf. Intell. Trans. Syst., 2002, pp. 926-931.

[3] N. Patwari, J. N. Ash, S. Kyperountas, A. O. Hero, III, R. L. Moses, and N. S. Correal, "Locating the nodes: Cooperative localization in wireless sensor networks," IEEE Signal Process. Mag., vol. 22, no. 4, pp. 54-69, Jul. 2005.

[4] Y. Zhao, "Standardization of mobile phone positioning for 3G systems," IEEE Commun. Mag., vol. 40, no. 7, pp. 108-116, Jul. 2002.

[5] A. H. Sayed, A. Tarighat, and N. Khajehnouri, "Network-based wireless location: Challenges faced in developing techniques for accurate wireless location information," IEEE Signal Process. Mag., vol. 22, no. 4, pp. 2540, Jul. 2005.

[6] S. Gezici, Z. Tian, G. B. Giannakis, H. Kobayashi, A. F. Molisch, H. V. Poor, and Z. Sahinoglu, "Localization via ultrawideband radios: A look at positioning aspects for future sensor network," IEEE Signal Process. Mag., vol. 22, no. 4, pp. 70-84, Jul. 2005.

[7] Y. T. Chan, C. H. Yau, and P. C. Yau, "Linear and approximate maximum likelihood localization from TOA measurements," in Proc. IEEE 7th Int. Symp. Signal Process. Appl., Jul. 2003, vol. 2, pp. 295-298.

[8] X. Wang, Z. Wang, and B. O'Dea, "TOA-based location algorithm reducing the errors due to non-line-of-sight (NLOS) propagation," IEEE Trans. Veh. Technol., vol. 52, no. 1, pp. 112-116, Jan. 2003.
[9] D. Porcino, "Performance of a OTDOA-IPDL positioning receiver for 3GPP-FDD mode," in Proc. IEE $3 G$ Mobile Commun. Technol., Mar. 2001, pp. 221-225.

[10] Location Technologies for GSM, GPRS, and UMTS Network, Campbell, CA: SnapTrack Inc. [Online]. Available: http://www.snaptrack.com/

[11] GPS Guide for Beginners, Garmin Corp., Olathe, KS. [Online]. Available: http://www.garmin.com/aboutGPS/manual.html

[12] K. Wang, L. Yan, H. Wen, and K. He, "GpsOne: A new solution to vehicle navigation," in Proc. IEEE Position Location Navigat. Symp., Apr. 2004, pp. 341-346.

[13] Y. T. Chen and K. C. Ho, "A simple and efficient estimator for hyperbolic location," IEEE Trans. Signal Process., vol. 42, no. 8, pp. 1905-1915, Aug. 1994.

[14] G. R. Iverson, Bayesian Statistical Inference. Beverly Hills, CA: Sage, 1984.

[15] K. Ostmann and A. E. Bell, "A data fusion architecture for enhanced position estimation in wireless networks," IEEE Commun. Lett., vol. 5, no. 8, pp. 343-345, Aug. 2001.

[16] B. L. Le, K. Ahmed, and H. Tsuji, "Mobile location estimator with NLOS mitigation using Kalman filtering," in Proc. IEEE Wireless Commun. Netw. Conf., Mar. 2003, vol. 3, pp. 1969-1973.

[17] "Third Generation Partnership Project (3GPP) Specifications Release 7,” 3GPP TS 21.101. [Online]. Available: http://www.3gpp. org/specs/releases.htm

[18] Draft Standard for Local and Metropolitan Area Networks Part 16: Air Interface for Broadband Wireless Access Systems, IEEE Std. 802.16 Rev3 (Revision of IEEE Std. 802.16-2004), 2008.

[19] J. Chaffee and J. Abel, "GDOP and the Cramér-Rao bound," in Proc. IEEE Position Location Navigat. Symp., Apr. 1994, pp. 663-668.

[20] L. Cong and W. Zhuang, "Non-line-of-sight error mitigation in mobile location," IEEE Trans. Wireless Commun., vol. 4, no. 2, pp. 560-573, Mar. 2005.

[21] Y. Qi, H. Kobayashi, and H. Suda, "Analysis of wireless geolocation in a non-line-of-sight environment," IEEE Trans. Wireless Commun., vol. 5, no. 3, pp. 672-681, Mar. 2006.

[22] Y.-T. Chan, W.-Y. Tsui, H.-C. So, and P.-C. Ching, "Time-of-arrival-based localization under NLOS conditions," IEEE Trans. Veh. Technol., vol. 55, no. 1, pp. 17-23, Jan. 2006.

[23] L. M. Kaplan, "Global node selection for localization in a distributed sensor network," IEEE Trans. Aerosp. Electron. Syst., vol. 42, no. 1, pp. 114-135, Jan. 2006.

[24] L. M. Kaplan, "Local node selection for localization in a distributed sensor network," IEEE Trans. Aerosp. Electron. Syst., vol. 42, no. 1, pp. 136-146, Jan. 2006.

[25] J. Liu, M. Lu, X. Cui, and Z. Feng, "Theoretical analysis of RAIM in the occurrence of simultaneous two-satellite faults," IET Trans. Radar Sonar Navig., vol. 1, no. 2, pp. 92-97, Apr. 2007.

[26] J. B.-Y. Tsui, Fundamentals of Global Positioning System Receivers: A Software Approach. Hoboken, NJ: Wiley.

[27] K.-T. Feng, C.-L. Chen, and C.-H. Chen, "GALE: An enhanced geometryassisted location estimation algorithm for NLOS environments," IEEE Trans. Mobile Comput., vol. 7, no. 2, pp. 199-213, Feb. 2008.

[28] Y. Yong and M. Lingjuan, "GDOP results in all-in-view positioning and in four optimum satellites positioning with GPS PRN codes ranging," in Proc. IEEE Position Location Navigat. Symp., Apr. 2004, pp. 723-727. 
[29] N. Levanon, "Lowest GDOP in 2-D scenarios," Proc. Inst. Elect. Eng.-Radar, Sonar Navig., vol. 147, no. 3, pp. 149-155, Jun. 2000.

[30] B. S. Pervan, D. G. Lawrence, C. E. Cohen, and B. W. Parkinson, "Parity space methods for autonomous fault detection and exclusion using GPS carrier phase," in Proc. IEEE Position Location Navigat. Symp., Apr. 1996, pp. 649-656.

[31] M. S. Phatak, "Recursive method for optimum GPS satellite selection," IEEE Trans. Aerosp. Electron. Syst., vol. 37, no. 2, pp. 751-754, Apr. 2001.

[32] S. A. Jazzar, J. Caffery, Jr., and H. R. You, "A scattering-model-based approach to NLOS mitigation in TOA location systems," in Proc. IEEE Veh. Technol. Conf., May 2002, pp. 861-865.

[33] S.-S. Woo, H.-R. You, and J.-S. Koh, "The NLOS mitigation technique for position location using IS-95 CDMA networks," in Proc. IEEE Veh. Technol. Conf., Sep. 2000, pp. 2556-2560.

[34] P. C. Chen, "A non-line-of-sight error mitigation algorithm in location estimation," in Proc. IEEE Wireless Commun. Netw. Conf., Sep. 1999, pp. 316-320.

[35] L. Cong and W. Zhuang, "Hybrid TDOA/AOA mobile user location for wideband CDMA cellular systems," IEEE Trans. Wireless Commun., vol. 1, no. 3, pp. 439-447, Jul. 2002.

[36] T. H. Cormen, C. E. Leiserson, R. L. Rivest, and C. Stein, Introduction to Algorithms. Cambridge, MA: MIT Press, 2001.

[37] M. J. Goris, D. A. Gray, and I. M. Y. Mareels, "Reducing the computational load of a Kalman filter," Electron. Lett., vol. 33, no. 18, pp. 15391541, Aug. 1997.

[38] H. Kuusniemi, A. Wieser, G. Lachapellea, and J. Takala, "User-level reliability monitoring in urban personal satellite navigation," IEEE Trans. Aerosp. Electron. Syst., vol. 43, no. 4, pp. 1305-1318, Oct. 2007.

[39] C. Y. Lee, Mobile Communications Engineering. New York: McGraw-Hill, 1997.

[40] Satscape Homepage: Freeware Satellite Tracker. [Online]. Available: http://www.satscape.co.uk

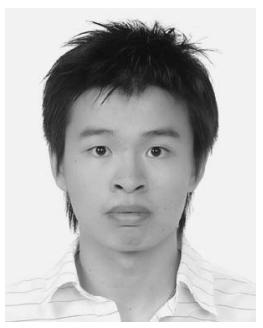

Po-Hsuan Tseng (S'08) received the B.S. degree in communications engineering in 2005 from the National Chiao Tung University, Hsinchu, Taiwan, where he is currently working toward the Ph.D. degree with the Department of Communications Engineering.

His research interests include wireless location technologies and mobile WiMax system design.

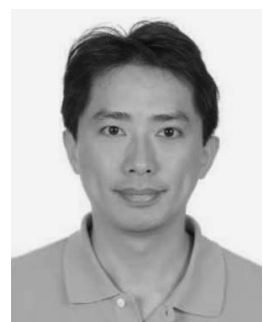

Kai-Ten Feng (M'03) received the B.S. degree from the National Taiwan University, Taipei, Taiwan, in 1992, the MS degree from the University of Michigan, Ann Arbor, in 1996, and the Ph.D. degree from the University of California, Berkeley, in 2000 .

From 2000 to 2003, he was an In-Vehicle Development Manager/Senior Technologist with the OnStar Corporation USA, which is a subsidiary of the General Motors Corporation, working on the design of future telematics platforms and in-vehicle networks. From February 2003 to July 2007, he was an Assistant Professor with the Department of Communications Engineering, National Chiao Tung University, Hsinchu, Taiwan, where he has been an Associate Professor since August 2007. His research interests include cooperative and cognitive networks, mobile ad hoc and sensor networks, embedded system design, wireless location technologies, and intelligent transportation systems.

Dr. Feng has served on the Technical Program Committees of the IEEE Vehicular Technology Conference, the IEEE International Conference on Communications, and the IEEE Asia-Pacific Wireless Communications Symposium. He was the recipient of the Best Paper Award at the Spring 2006 IEEE Vehicular Technology Conference, for which his paper ranked the first among the 615 accepted papers, and the 2007 Outstanding Young Electrical Engineer Award from the Chinese Institute of Electrical Engineering. 